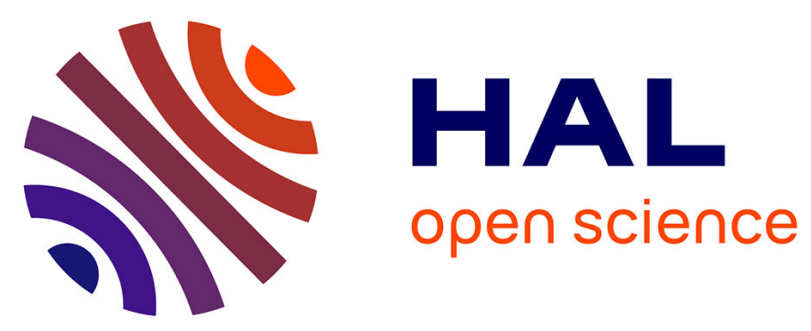

\title{
A physically-based model for retrieving foliar biochemistry and leaf orientation using close-range imaging spectroscopy
}

S. Jay, R. Bendoula, X. Hadoux, J.B. Féret, N. Gorretta

\section{- To cite this version:}

S. Jay, R. Bendoula, X. Hadoux, J.B. Féret, N. Gorretta. A physically-based model for retrieving foliar biochemistry and leaf orientation using close-range imaging spectroscopy. Remote Sensing of Environment, 2016, 177, pp.220-236. 10.1016/j.rse.2016.02.029 . hal-01804958

\section{HAL Id: hal-01804958 \\ https://hal.science/hal-01804958}

Submitted on 1 Jun 2018

HAL is a multi-disciplinary open access archive for the deposit and dissemination of scientific research documents, whether they are published or not. The documents may come from teaching and research institutions in France or abroad, or from public or private research centers.
L'archive ouverte pluridisciplinaire HAL, est destinée au dépôt et à la diffusion de documents scientifiques de niveau recherche, publiés ou non, émanant des établissements d'enseignement et de recherche français ou étrangers, des laboratoires publics ou privés. 


\title{
A physically-based model for retrieving foliar biochemistry and leaf orientation using close-range imaging spectroscopy
}

\author{
Sylvain Jay ${ }^{\mathrm{a}, *}$, Ryad Bendoula ${ }^{\mathrm{a}}$, Xavier Hadoux ${ }^{\mathrm{b}, \mathrm{c}}$, Jean-Baptiste Féret ${ }^{\mathrm{d}}$, \\ Nathalie Gorretta ${ }^{\mathrm{a}}$ \\ ${ }^{a}$ Irstea, UMR ITAP, 361 rue J.F. Breton, 34196 Montpellier, France \\ ${ }^{b}$ Centre for Eye Research Australia, Royal Victorian Eye and Ear Hospital, Melbourne, \\ Australia \\ ${ }^{c}$ Ophthalmology, University of Melbourne, Department of Surgery, Melbourne, Australia \\ ${ }^{d}$ Irstea, UMR TETIS, Maison de la Télédétection, 500 rue J.F. Breton, 34093 \\ Montpellier, France
}

\begin{abstract}
Radiative transfer models have long been used to characterize the foliar content at the leaf and canopy levels. However, they still do not apply well to close-range imaging spectroscopy, especially because directional effects are usually not taken into account. For this purpose, we introduce a physical approach to describe and simulate the variation in leaf reflectance observed at this scale. Two parameters are thus introduced to represent (1) specular reflection at the leaf surface and (2) local leaf orientation. The model, called COSINE (ClOse-range Spectral ImagiNg of lEaves), can be coupled with a directional-hemispherical reflectance model of leaf optical properties to relate

*Corresponding author at: Irstea, UMR ITAP, 361 rue J.F. Breton, 34196 Montpellier, France. Tel: +33467166459 .

E-mail addresses: sylvain.jay@irstea.fr (S. Jay), ryad.bendoula@irstea.fr (R. Bendoula), xavier@hadoux.com (X. Hadoux), jean-baptiste.feret@teledetection.fr (J.B. Féret), nathalie.gorretta@irstea.fr (N. Gorretta).
\end{abstract}


the measured reflectance to the foliar content. In this study, we show that, when combining COSINE with the PROSPECT model, the overall PROCOSINE model allows for a robust sub-millimeter retrieval of foliar content based on numerical inversion and pseudo bidirectional reflectance factor hyperspectral measurements.

The relevance of the added parameters is first shown through a sensitivity analysis performed in the visible and near-infrared (VNIR) and shortwave infrared (SWIR) ranges. PROCOSINE is then validated based on VNIR and SWIR hyperspectral images of various leaf species exhibiting different surface properties. Introducing these two parameters within the inversion allows us to obtain accurate maps of PROSPECT parameters, e.g., the chlorophyll content in the VNIR range, and the equivalent water thickness and leaf mass per area in the SWIR range. Through the estimation of light incident angle, the PROCOSINE inversion also provides information on leaf orientation, which is a critical parameter in vegetation remote sensing.

Keywords: close-range, COSINE, hyperspectral, imaging spectroscopy, leaf optical properties, pigment retrieval, PROCOSINE, PROSPECT, radiative transfer, vegetation

\section{Introduction}

Due to the strong interactions occurring between vegetation and the in-

3 coming optical radiation through absorption and scattering processes, hyper-

4 spectral remote sensing from satellites and aircrafts provides critical infor-

5 mation to assess the spatial and temporal variabilities of vegetation status 6 from local to global scales. This has led to a number of agricultural, envi- 
ronmental and ecological applications such as the retrieval of leaf pigments (Zarco-Tejada et al., 2004; Ustin et al., 2009), the early detection of leaf diseases (Mahlein et al., 2013) or the mapping of forest biodiversity (Féret \& Asner, 2014). As hyperspectral cameras are now becoming more affordable, close-range remote sensing data are also increasingly available to the scientific community. Compared with air- and satellite-borne data, they generally offer a sub-milliter or millimeter spatial resolution, and they can be acquired at a higher temporal frequency, which is particularly interesting for precision agriculture. For example, these data can be used to identify plant pigments (Blackburn, 2007), freezing stress (Nicotra et al., 2003) or leaf diseases (Mahlein et al., 2013), each of which is of tremendous importance to follow up the plant physiological status. These images are generally processed by applying statistically-based methods to estimate various leaf biochemical properties (Nicotra et al., 2003; Vigneau et al., 2011; Ji-Yong et al., 2012; Jay et al., 2014). However, at this scale, a proper physical interpretation based on radiative transfer modeling is needed to describe the interactions between light and vegetation, especially for a spatially- and temporally-resolved quantification of pigments (Blackburn, 2007).

Vegetation radiative transfer models are physically-based and simulate light propagation within leaves and/or canopies, e.g., as a function of leaf biochemical constituents, leaf anatomy or canopy structure. Whenever possible, model inversion allows for the retrieval of the variables of interest, generally using iterative optimization, look-up tables, statistical methods or machine learning algorithms. 
At the leaf level, these models range from simple plate models, to ray-tracing, radiosity and stochastic models that are computationally more difficult to invert (Dorigo et al., 2007). For instance, PROSPECT (Jacquemoud \& Baret, 1990) is based on the generalized plate model, and is particularly well suited to estimate leaf biochemical constituents (e.g., chlorophyll content, water content and leaf mass per area) based on spectral measurements in the optical domain. The main reasons for the popularity of PROSPECT are its accuracy, its computational efficiency (resulting in fast iterative model inversion) and free distribution.

At the canopy level, various approaches of different complexities have been developed for radiative transfer modeling, e.g., turbid medium approaches (Verhoef, 1984), geometrical approaches (Chen \& Leblanc, 1997) or the combination of both (Gastellu-Etchegorry et al., 1996). Most of these models allow the canopy reflectance to be modeled as a function of parameters related to canopy structure (such as leaf area index or leaf inclination distribution function), leaf optical properties and sun-sensor geometry.

However, leaf and canopy radiative transfer models do not apply well to close-range imaging spectroscopy. For example, at the leaf level, the directional-hemispherical reflectance and transmittance simulated by PROSPECT (Jacquemoud \& Baret, 1990) are usually measured with an integrating sphere, whose implementation is difficult (if not impossible) for every single pixel of hyperspectral images. As a result, PROSPECT cannot be inverted based on directional reflectance data as retrieved by a close-range hyperspectral camera, unless it is assumed that leaves are Lambertian (Buddenbaum \& Hill, 
2015) and in fully horizontal position, which is an unrealistic hypothesis. Indeed, in most cases, leaf reflectance exhibits some anisotropy (Bousquet et al., 2005; Comar et al., 2012) and thus varies with respect to illumination and viewing angles. Furthermore, variation in leaf orientation prevent from achieving a proper reflectance correction for every pixel, because the reference surface used for reflectance correction is generally not submitted to the same local illumination conditions than leaf material.

At the canopy level, most radiative transfer models have to be applied to mixed pixels (containing both soil and leaf materials), for which effects of leaf composition, canopy structure, soil properties and viewing/illumination angles are integrated into a single spectrum. Canopy models are thus well suited for ground-based spectroradiometric measurements, as well as for airand satellite-borne hyperspectral measurements, all of them being usually characterized by a spatial resolution coarser than one meter (Zarco-Tejada et al., 2003; Colombo et al., 2008; Schlemmer et al., 2013). However, most canopy models are not suitable for simulating hyperspectral data characterized by a higher spatial resolution (up to sub-millimeter level) for which the assumption of mixed pixel does not hold.

In this study, we propose a physically-based model, called COSINE (ClOserange Spectral ImagiNg of lEaves), that describes the additional spectral variability induced by directional effects and variation in leaf orientation. Combining COSINE with a leaf directional-hemispherical reflectance model such as PROSPECT allows the simulation of leaf reflectance according to our experimental conditions: submillimetric spatial resolution and a single 
light source assumed to be directional. When applied in inverse mode to close-range hyperspectral images, the overall PROCOSINE model enables the simultaneous retrieval of PROSPECT parameters (e.g., chlorophyll and water contents), bidirectional effects and leaf angle with respect to the light source.

The COSINE theory is described in Section 2. After recalling the necessary radiometric definitions, we develop a physically-based analytic expression of the reflectance quantity retrieved using close-range imaging spectroscopy. This expression is then related to PROSPECT to explain variations in leaf biochemistry and leaf anatomy. In Section 3, we present the data sets used in this article as well as details about model validation and sensitivity analysis. Results are presented and discussed in Section 4, and we finally draw some conclusions and perspectives in Section 5.

\section{Theory}

\subsection{Radiometric considerations}

\subsubsection{Definitions}

The definitions and notations of the main physical quantities used in this article and summarized in Table 1, are based on the initial terminology of Nicodemus et al. (1977), which has later been reviewed by Schaepman-Strub et al. (2006).

The spectral radiance $L$ is the radiant flux in a beam per unit wavelength, per unit area and per unit solid angle, and is expressed in the SI unit $\left[\mathrm{W} \cdot \mathrm{sr}^{-1} \cdot \mathrm{m}^{-2} \cdot \mathrm{nm}^{-1}\right]$. This is the physical quantity measured by a hyper- 
Table 1: Main parameters and acronyms.

\begin{tabular}{|c|c|}
\hline Parameter & Definition [unit] \\
\hline$b_{\text {spec }}$ & Specular term [unitless] \\
\hline$C_{a b}$ & Chlorophyll a+b content $\left[\mu \mathrm{g} . \mathrm{cm}^{-2}\right]$ \\
\hline$C_{b p}$ & Brown pigment content [unitless] \\
\hline$C_{c x}$ & Carotenoid content $\left[\mu \mathrm{g} . \mathrm{cm}^{-2}\right]$ \\
\hline$C_{m}$ & Leaf mass per area $\left[\mathrm{g} . \mathrm{cm}^{-2}\right]$ \\
\hline$C_{w}$ & Equivalent water thickness $[\mathrm{cm}]$ \\
\hline$E$ & Spectral irradiance $\left[\mathrm{W} \cdot \mathrm{m}^{-2} \cdot \mathrm{nm}^{-1}\right]$ \\
\hline$f_{r}$ & Bidirectional reflectance distribution function (BRDF) $\left[\mathrm{sr}^{-1}\right]$ \\
\hline$L$ & Spectral radiance $\left[\mathrm{W} \cdot \mathrm{sr}^{-1} \cdot \mathrm{m}^{-2} \cdot \mathrm{nm}^{-1}\right]$ \\
\hline$\lambda$ & Wavelength $[\mathrm{nm}]$ \\
\hline$N$ & Leaf structure parameter [unitless] \\
\hline$\varphi_{l}$ & Difference between illumination and leaf normal azimuth angles $\left[^{\circ}\right]$ \\
\hline$\varphi_{v}$ & Difference between illumination and viewing azimuth angles $\left[^{\circ}\right]$ \\
\hline$R$ & Bidirectional reflectance factor (BRF) [unitless] \\
\hline$R_{h s i}$ & Pseudo bidirectional reflectance factor [unitless] \\
\hline$\rho$ & Directional-hemispherical reflectance (DHR) [unitless] \\
\hline$\theta_{i}$ & $\begin{array}{l}\text { Light incident angle (angle between the light source and the normal } \\
\text { to the leaf) }\left[^{\circ}\right]\end{array}$ \\
\hline$\theta_{l}, \theta_{v}, \theta_{s}$ & Leaf normal, viewing and illumination zenith angles $\left[^{\circ}\right]$ \\
\hline$\vartheta$ & PROCOSINE parameters \\
\hline$\vartheta_{d h r}$ & Parameters of the leaf DHR model \\
\hline
\end{tabular}


spectral imaging sensor after spectral calibration. The spectral irradiance $E$ is the radiant flux in a beam per unit wavelength and per unit area and is expressed in $\left[\mathrm{W} \cdot \mathrm{m}^{-2} \cdot \mathrm{nm}^{-1}\right]$.

One of the main physical quantities used to describe angular patterns of reflected light is the bidirectional reflectance distribution function (BRDF) expressed in $\left[\mathrm{sr}^{-1}\right]$. It describes how a parallel beam of incident light from one direction in the hemisphere is reflected into another direction in the hemisphere:

$$
f_{r}\left(\theta_{s} ; \theta_{v}, \varphi_{v} ; \lambda\right)=\frac{d L_{r}\left(\theta_{s} ; \theta_{v}, \varphi_{v} ; \lambda\right)}{d E_{i}\left(\theta_{s} ; \lambda\right)}
$$

where subscripts $i$ and $r$ refer to incoming and reflected lights respectively, $\theta_{s}$ and $\theta_{v}$ are respectively the illumination and viewing zenith angles, and $\varphi_{v}$ is the viewing azimuth angle relatively to the illumination azimuth angle (see Fig. 1 for angle representation). The BRDF being the ratio of two infinitesimal quantities, it cannot theoretically be measured. However, its integration over the corresponding solid angles allows the derivation of many other measurable physical quantities.

Usually, the reflectance correction process does not consist in retrieving directly the reflectance (defined as the ratio of the leaving radiant exitance to the incident irradiance), but rather follows the definition of a reflectance factor. In the specific case of single illumination and viewing directions, the bidirectional reflectance factor (BRF, denoted by $R$ ) is given by the ratio of the radiant flux $d L_{r}$ reflected from the area element $d A$ to the radiant flux $d L_{r}^{i d}$ reflected from an ideal and diffuse surface of the same area $d A$ under identical illumination and viewing geometries. It is unitless and, as developed 


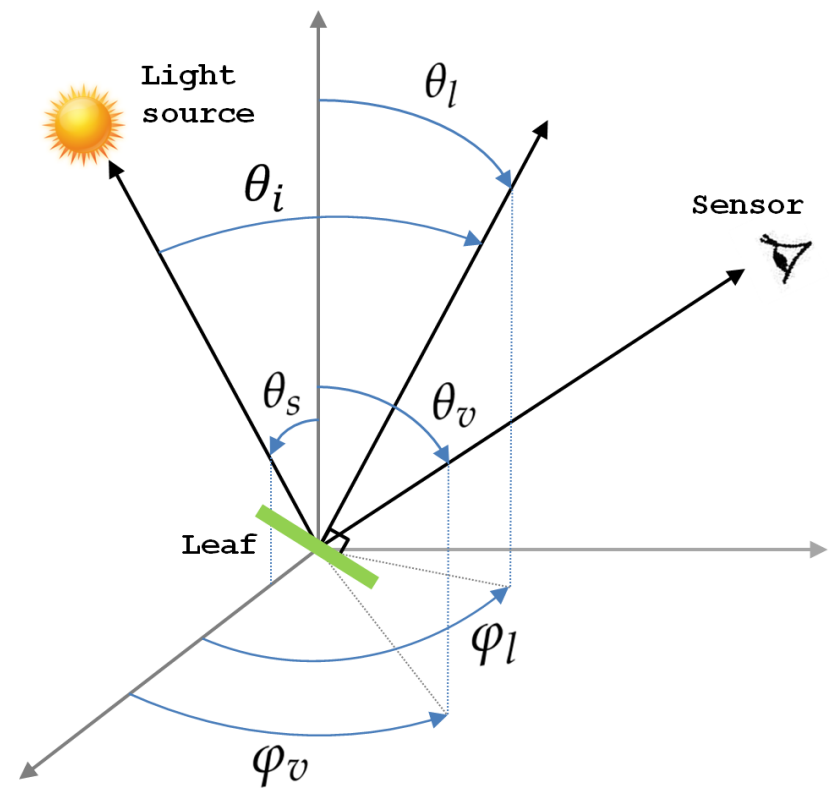

Figure 1: Angle representation.

by Schaepman-Strub et al. (2006), it can be expressed as follows:

$$
R\left(\theta_{s} ; \theta_{v}, \varphi_{v} ; \lambda\right)=\frac{d L_{r}\left(\theta_{s} ; \theta_{v}, \varphi_{v} ; \lambda\right)}{d L_{r}^{i d}\left(\theta_{s} ; \lambda\right)}
$$

where $d L_{r}^{i d}$ does not depend on viewing angles because of the Lambertian nature of the reference surface.

The BRDF of an ideal and diffuse surface being $(1 / \pi)$, the BRF of any surface is therefore related to its BRDF by:

$$
R\left(\theta_{s} ; \theta_{v}, \varphi_{v} ; \lambda\right)=\pi f_{r}\left(\theta_{s} ; \theta_{v}, \varphi_{v} ; \lambda\right)
$$

(3)

6

Let us finally define the directional-hemispherical reflectance (DHR) as the integration of BRDF over the whole viewing hemisphere. The DHR is unitless and is given by:

$$
\rho\left(\theta_{s} ; 2 \pi ; \lambda\right)=\int_{0}^{2 \pi} \int_{0}^{\pi / 2} f_{r}\left(\theta_{s} ; \theta_{v}, \varphi_{v} ; \lambda\right) \cos \theta_{v} \sin \theta_{v} d \theta_{v} d \varphi_{v} .
$$


In the following, the spectral dependence will be omitted for more clarity in notation. Similarly, spectral radiance and spectral irradiance will be simply referred to as radiance and irradiance.

\subsubsection{Radiometric expression of close-range hyperspectral measurement}

The solid angles corresponding to incident and reflected light beams are never purely directional, so rigorously, sensor measurements only allow the retrieval of a biconical reflectance factor (Schaepman-Strub et al., 2006). However, a close approximation of bidirectional reflectance can be obtained under specific experimental and instrumental conditions, e.g., using a collimated light beam and a small sensor field of view (FOV) provided by a push-broom hyperspectral camera. In this case, the leaf BRF $R$ is approximated as follows:

$$
R\left(\theta_{s} ; \theta_{v}, \varphi_{v}\right) \approx \frac{L_{r}\left(\theta_{s} ; \theta_{v}, \varphi_{v}\right)}{L_{r}^{i d}\left(\theta_{s}\right)}
$$

where $L_{r}$ and $L_{r}^{i d}$ are the radiances respectively measured on the leaf and on the reference surface with a hyperspectral camera.

Importantly, Eq. 5 requires the leaf and reference surface to be under identical illumination geometry (i.e., same $\theta_{s}$ values). However, at the pixel level, the leaf position and leaf local orientation can make the irradiances received by the leaf and reference surface strongly different. Indeed, considering a directional light source, the irradiance received by an area element is proportional to the cosine of the incident angle $\theta_{i}$, i.e., the angle between the light source and the normal to this element:

$$
E_{i}\left(\theta_{i}\right)=E_{0} \cos \theta_{i}
$$


where $E_{0}$ is the irradiance received by an area element perpendicular to the light source direction, and $\cos \theta_{i}=\cos \theta_{s} \cos \theta_{l}+\sin \theta_{s} \sin \theta_{l} \cos \varphi_{l}$ where $\theta_{l}$ and $\varphi_{l}$ characterize the normal to this area element (Bousquet et al., 2005; Comar et al., 2014).

Therefore, we define two illumination geometries corresponding to either the leaf or the reference surface. The irradiance received by the leaf is:

$$
E_{i}^{l}\left(\theta_{i}^{l}\right)=E_{0} \cos \theta_{i}^{l}
$$

whereas the one received by the ideal and diffuse reference surface is:

$$
E_{i}^{i d}\left(\theta_{i}^{i d}\right)=E_{0} \cos \theta_{i}^{i d} .
$$

The reference surface is usually positioned horizontally so, in the following, we note $\theta_{i}^{i d}=\theta_{s}$ and $\theta_{i}^{l}=\theta_{i}$.

Similarly to Eq. 5, at each pixel, the physical quantity $R_{h s i}$ retrieved using a hyperspectral camera is given by:

$$
R_{h s i}\left(\theta_{s}, \theta_{i} ; \theta_{v}, \varphi_{v}\right) \approx \frac{L_{r}\left(\theta_{i} ; \theta_{v}, \varphi_{v}\right)}{L_{r}^{i d}\left(\theta_{s}\right)} .
$$

Assuming the light source is directional and the pixel FOV is small, combining Eq. 1 and Eq. 9 leads to the following equation:

$$
R_{h s i}\left(\theta_{s}, \theta_{i} ; \theta_{v}, \varphi_{v}\right) \approx \frac{f_{r}\left(\theta_{i} ; \theta_{v}, \varphi_{v}\right) E_{i}^{l}\left(\theta_{i}\right)}{(1 / \pi) E_{i}^{i d}\left(\theta_{s}\right)} .
$$

Using Eq. 3, Eq. 7 and Eq. 8, $R_{h s i}$ can finally be expressed as follows:

$$
R_{h s i}\left(\theta_{s}, \theta_{i} ; \theta_{v}, \varphi_{v}\right) \approx R\left(\theta_{i} ; \theta_{v}, \varphi_{v}\right) \frac{\cos \theta_{i}}{\cos \theta_{s}}
$$

Eq. 11 reveals that the physical reflectance quantity retrieved from a small FOV sensor in presence of a directional light source does not correspond 
to the leaf BRF if the leaf and reference surface are differently tilted with respect to the light source. In the following, we call "leaf pseudo BRF" the leaf BRF weighted by the ratio of the cosine of the angle between the light source and the normal to the leaf, to the cosine of illumination zenith angle. Therefore, Eq. 11 relates the measured leaf pseudo BRF to the illumination zenith angle, the incident angle (related to local leaf angles and illumination zenith angle) and the leaf BRF. To relate the latter to the foliar content, it is then necessary to take into account leaf surface properties as described in the next section.

\subsection{COSINE: a model for ClOse-range Spectral ImagiNg of lEaves}

In this study, we propose to adapt a leaf DHR model (such as PROSPECT (Jacquemoud \& Baret, 1990) or LIBERTY (Dawson et al., 1998)) to the closerange case. Indeed, with the perspective of comparing leaf optical measurements to DHR simulations, proper experimental acquisitions require using an integrating sphere in order to match with the hemispherical simulations. As a result, leaf DHR models cannot directly be applied to hyperspectral remote sensing observations of vegetation that are affected by variable bidirectional effects (or BRDF effects) depending on illumination and viewing geometries. An important prerequisite before using these models for close-range imaging spectroscopy, is therefore to relate the leaf DHR to the leaf BRDF.

\subsubsection{Relationship between leaf BRDF and DHR}

It is generally admitted that the leaf BRDF is the sum of a diffuse component $f_{r, \text { diff }}$ and a directional component $f_{r, s p e c}$ (Bousquet et al., 2005; Comar 
et al., 2014):

$$
f_{r}\left(\theta_{i} ; \theta_{v}, \varphi_{v}\right)=f_{r, s p e c}\left(\theta_{i} ; \theta_{v}, \varphi_{v}\right)+f_{r, d i f f}\left(\theta_{i} ; \theta_{v}, \varphi_{v}\right) .
$$

The diffuse component $f_{r, \text { diff }}$ characterizes absorption and scattering processes within the leaf volume and thus depends on optically-active biochemical and biophysical parameters. The directional component $f_{r, s p e c}$ characterizes leaf surface properties and describes how light is reflected at the surface. While $f_{r, d i f f}$ is strongly wavelength-dependent, $f_{r, s p e c}$ is assumed to be nearly wavelength-independent in the visible and near-infrared (VNIR) range (between 400 and $1000 \mathrm{~nm}$ ) (Bousquet et al., 2005; Comar et al., 2014). In the shortwave infrared (SWIR) range (between 1000 and $2500 \mathrm{~nm}$ ), this assumption does not hold because the leaf refractive index actually depends on wavelength (Féret et al., 2008; Vanderbilt \& Grant, 1985), especially due to the non-negligible influence of water absorption.

As a first approximation, $f_{r, d i f f}$ can be expressed as the ratio of leaf mesophyll Lambert coefficient $k_{l}$ to $\pi$ (Bousquet et al., 2005). To take into account the two-layer leaf structure (i.e. composed of an upper wax layer and a bottom leaf mesophyll layer), one should also consider the fraction of light that is reflected by the first wax layer and that does not reach the bottom layer (Stuckens et al., 2009; Ashikmin et al., 2000). The diffuse component is then expressed as a function of leaf mesophyll Lambert coefficient $k_{l}$ and wax DHR $\rho_{\text {spec }}$ (Stuckens et al., 2009):

$$
f_{r, d i f f}\left(\theta_{i}\right)=\frac{k_{l}}{\pi}\left(1-\rho_{\text {spec }}\left(\theta_{i} ; 2 \pi\right)\right)
$$

where $f_{r, \text { diff }}$ does not depend on viewing angles and $k_{l}$ only depends on 
wavelength.

As defined by Eq. 4, the total DHR can then be obtained combining Eq. 12 and Eq. 13:

$$
\begin{aligned}
\rho\left(\theta_{i} ; 2 \pi\right)=\int_{0}^{2 \pi} \int_{0}^{\pi / 2} f_{r, \text { spec }}\left(\theta_{i} ; \theta_{v}, \varphi_{v}\right) \cos \theta_{v} \sin \theta_{v} d \theta_{v} d \varphi_{v} \\
+\frac{k_{l}}{\pi}\left(1-\rho_{\text {spec }}\left(\theta_{i} ; 2 \pi\right)\right) \int_{0}^{2 \pi} \int_{0}^{\pi / 2} \cos \theta_{v} \sin \theta_{v} d \theta_{v} d \varphi_{v}
\end{aligned}
$$

which can be rewritten as follows:

$$
\rho\left(\theta_{i} ; 2 \pi\right)=\rho_{\text {spec }}\left(\theta_{i} ; 2 \pi\right)+k_{l}\left(1-\rho_{\text {spec }}\left(\theta_{i} ; 2 \pi\right)\right) .
$$

This equation provides the necessary basis to relate a leaf DHR model to the leaf BRDF (through the Lambert coefficient).

\subsubsection{The COSINE model}

Assuming nadir illumination, a leaf DHR model expresses the DHR $\rho(0 ; 2 \pi)$ as a function of leaf biochemical and biophysical contents characterized by the vector of input parameters $\boldsymbol{\vartheta}_{d h r}$. Following Eq. 15, the modeled Lambert coefficient can be expressed as a function of $\rho_{\text {spec }}(0 ; 2 \pi)$ and DHR model $\widetilde{\rho}\left(\boldsymbol{\vartheta}_{d h r}\right)$ as:

$$
\widetilde{k}_{l}\left(\boldsymbol{\vartheta}_{d h r}\right)=\frac{\widetilde{\rho}\left(\boldsymbol{\vartheta}_{d h r}\right)-\rho_{\text {spec }}(0 ; 2 \pi)}{1-\rho_{\text {spec }}(0 ; 2 \pi)}
$$

where $\sim$ refers to modeled quantities.

The leaf BRDF can then be modeled as a function of leaf parameters $\boldsymbol{\vartheta}_{d h r}$ by combining Eq. 12, Eq. 13 and Eq. 16:

$$
\begin{aligned}
\widetilde{f}_{r}\left(\theta_{i} ; \theta_{v}, \varphi_{v} ; \boldsymbol{\vartheta}_{d h r}\right)= & f_{r, \text { spec }}\left(\theta_{i} ; \theta_{v}, \varphi_{v}\right) \\
& +\frac{1}{\pi}\left(\frac{1-\rho_{\text {spec }}\left(\theta_{i} ; 2 \pi\right)}{1-\rho_{\text {spec }}(0 ; 2 \pi)}\right)\left(\widetilde{\rho}\left(\boldsymbol{\vartheta}_{d h r}\right)-\rho_{\text {spec }}(0 ; 2 \pi)\right) .
\end{aligned}
$$


In order to estimate $\boldsymbol{\vartheta}_{d h r}$ from the pseudo BRF $R_{h s i}$ retrieved using a hyperspectral camera, Eq. 3, Eq. 11 and Eq. 17 are combined to obtain the pseudo-BRF based COSINE model:

$$
\begin{array}{r}
\widetilde{R_{h s i}}\left(\theta_{s}, \theta_{i} ; \theta_{v}, \varphi_{v} ; \boldsymbol{\vartheta}_{d h r}\right)=\left(\frac{\cos \theta_{i}}{\cos \theta_{s}}\right)\left[\left(\frac{1-\rho_{\text {spec }}\left(\theta_{i} ; 2 \pi\right)}{1-\rho_{\text {spec }}(0 ; 2 \pi)}\right) \widetilde{\rho}\left(\boldsymbol{\vartheta}_{\text {dhr }}\right)\right. \\
\left.+\pi f_{r, \text { spec }}\left(\theta_{i} ; \theta_{v}, \varphi_{v}\right)-\rho_{\text {spec }}(0 ; 2 \pi)\left(\frac{1-\rho_{\text {spec }}\left(\theta_{i} ; 2 \pi\right)}{1-\rho_{\text {spec }}(0 ; 2 \pi)}\right)\right]
\end{array}
$$

where only the first term of the sum is related to the leaf DHR model.

Note that $f_{r, \text { spec }}$ and $\rho_{\text {spec }}$ could potentially be modeled using the leaf BRDF model developed by Bousquet et al. (2005) that expresses the directional component as a function of illumination and viewing geometries as well as on the wax refractive index and a surface roughness parameter. Unfortunately, the resulting model becomes over-parameterized so its inversion is an ill-posed problem and leads to poor estimation results (not shown).

\subsubsection{A simplified COSINE model for the VNIR range}

In the VNIR range, the second term in Eq. 18 is assumed to be wavelengthindependent. Omitting its dependence in viewing angles, Eq. 18 can then be simplified as:

$$
\widetilde{R_{h s i}}\left(\theta_{s}, \theta_{i} ; \boldsymbol{\vartheta}_{d h r}, b_{\text {spec }}\right)=\left(\frac{\cos \theta_{i}}{\cos \theta_{s}}\right)\left[\left(\frac{1-\rho_{\text {spec }}\left(\theta_{i} ; 2 \pi\right)}{1-\rho_{\text {spec }}(0 ; 2 \pi)}\right) \widetilde{\rho}\left(\boldsymbol{\vartheta}_{d h r}\right)+b_{\text {spec }}\right]
$$

where $b_{\text {spec }}=b_{\text {spec }}\left(\theta_{i} ; \theta_{v}, \varphi_{v}\right)=\pi f_{r, \text { spec }}\left(\theta_{i} ; \theta_{v}, \varphi_{v}\right)-\rho_{\text {spec }}(0 ; 2 \pi)\left(\frac{1-\rho_{\text {spec }}\left(\theta_{i} ; 2 \pi\right)}{1-\rho_{\text {spec }}(0 ; 2 \pi)}\right)$ Assuming that the fraction $\frac{1-\rho_{\text {spec }}\left(\theta_{i} ; 2 \pi\right)}{1-\rho_{\text {spec }}(0 ; 2 \pi)}$ is nearly one (especially true for low incident angles), Eq. 19 can be approximated by:

$$
\widetilde{R_{h s i}}\left(\theta_{s}, \theta_{i} ; \boldsymbol{\vartheta}_{d h r}, b_{s p e c}\right)=\left(\frac{\cos \theta_{i}}{\cos \theta_{s}}\right)\left[\widetilde{\rho}\left(\boldsymbol{\vartheta}_{d h r}\right)+b_{\text {spec }}\right] \text {. }
$$


COSINE therefore simulates the pseudo-BRF as a function of incident angle $\theta_{i}$, illumination zenith angle $\theta_{s}$, foliar content through the DHR model parameters $\boldsymbol{\vartheta}_{d h r}$ and BRDF effect $b_{\text {spec }}$. Importantly, this model is presumably only well suited for low incident angles. In high incidence, the fraction $\frac{1-\rho_{\text {spec }}\left(\theta_{i} ; 2 \pi\right)}{1-\rho_{\text {spec }}(0 ; 2 \pi)}$ may differ from one, which could lead to an incorrect estimation of $\theta_{i}$ when inverting the model.

Finally, in order to model directly radiance measurements, a radiance-based COSINE model can be derived from Eq. 9 as:

$$
\widetilde{L_{r}}\left(\theta_{s}, \theta_{i} ; \boldsymbol{\vartheta}_{d h r}, b_{\text {spec }}\right)=\left(\frac{\cos \theta_{i}}{\cos \theta_{s}}\right)\left[\widetilde{\rho}\left(\boldsymbol{\vartheta}_{d h r}\right)+b_{s p e c}\right] L_{r}^{i d}\left(\theta_{s}\right)
$$

where $L_{r}^{i d}\left(\theta_{s}\right)$ is the radiance measured on the reference surface.

\section{Material and methods}

\subsection{Data acquisition}

\subsubsection{Spectral measurements}

The COSINE model was tested using close-range hyperspectral images acquired under laboratory conditions. Two push-broom hyperspectral cameras were used, i.e., a HySpex VNIR-1600 camera (Norsk Elektro Optikk, Norway) and a HySpex SWIR-320m-e camera (Norsk Elektro Optikk, Norway). The HySpex VNIR-1600 camera acquired successive lines of 1600 pixels and 160 spectral bands ranging from 415 to $994 \mathrm{~nm}$ with a $3.7 \mathrm{~nm}$ spectral sampling interval. The pixel FOV was 0.18 and $0.36 \mathrm{mrad}$ across- and alongtrack respectively.

Even if COSINE is theoretically only well suited for the VNIR range for which the directional BRDF component is assumed to be wavelength-independent, 
(a)

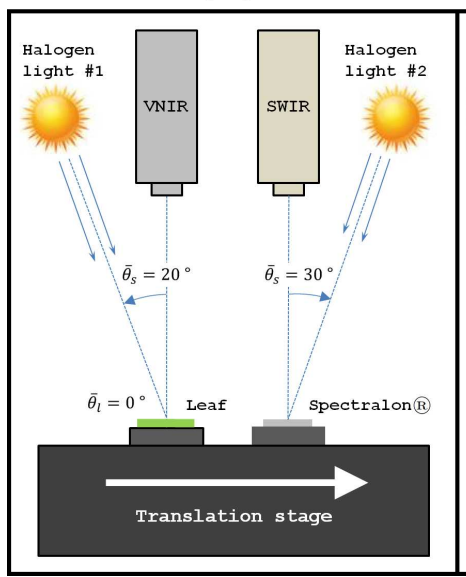

(b)

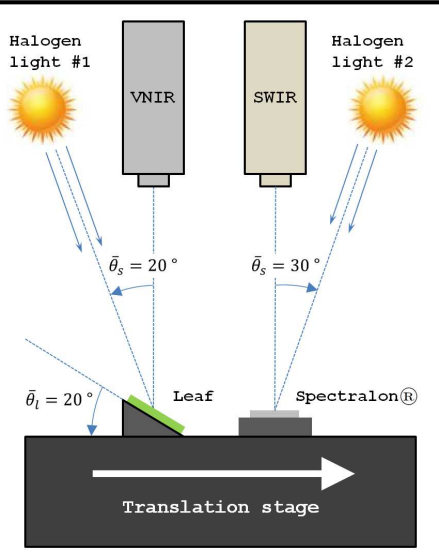

( c)

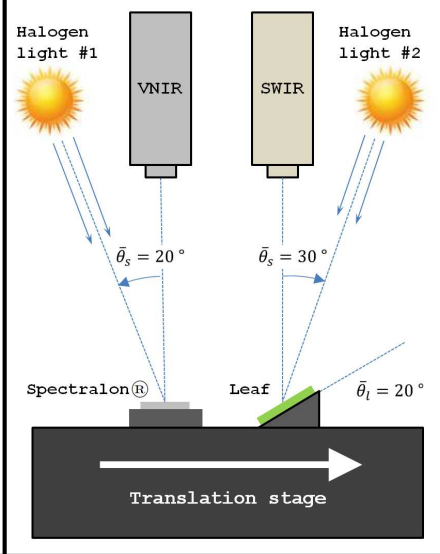

Figure 2: Experimental setup for the horizontal (a) and tilted (b-c) configurations. For the VNIR camera, the corresponding average incident angles $\bar{\theta}_{i}$ are $20^{\circ}(\mathrm{a}), 40^{\circ}$ (b) and $0^{\circ}$ (c). For the SWIR camera, the corresponding average incident angles $\bar{\theta}_{i}$ are $30^{\circ}$ (a), $10^{\circ}(\mathrm{b})$ and $50^{\circ}(\mathrm{c})$.

we also tested this model on SWIR images in order to see how this assumption was affecting the estimation results. The HySpex SWIR-320m-e camera acquired successive lines of 320 pixels and 256 spectral bands ranging from 960 to $2490 \mathrm{~nm}$ with a $6 \mathrm{~nm}$ spectral sampling interval. The pixel FOV was 0.75 mrad across- and along-track.

As shown in Fig. 2, in both cases, the camera was facing towards nadir at thirty centimeters above the imaged leaf, thus leading to across-track pixel FOV of $0.23 \mathrm{~mm}$ for the SWIR camera, and $0.11 \mathrm{~mm}$ for the VNIR camera (after an appropriate subsampling in the across-track direction so as to obtain square pixels). Both cameras were positioned thirty centimeters apart. The lighting was provided by two halogen sources (one for each camera) positioned close to the cameras. These light sources were collimated and 
positioned so that each source was illuminating the same line than the line imaged by the corresponding camera. The illumination zenith angles were set to $\theta_{s}=20^{\circ}$ and $\theta_{s}=30^{\circ}$ for the VNIR camera and the SWIR camera respectively. The incoming halogen irradiance was estimated on a line-byline basis by using a reference surface (Spectralon ${ }^{\circledR}$, Labsphere) horizontally placed next to the imaged leaf. In order to limit saturation on the reference surface while obtaining a low noise level in the strong absorption regions, we chose a Spectralon ${ }^{\circledR}$ whose diffuse reflectance was approximately $40 \%$ between 400 and $2500 \mathrm{~nm}$. After acquisition, VNIR and SWIR images were finally calibrated to spectral radiance.

The main data set contains leaves from five species commonly found in the French Mediterranean region, namely bamboo (Phyllostachys aurea), ivy (Hedera helix), laurestine (Viburnum tinus), bay laurel (Laurus nobilis) and holly (Ilex aquifolium). Some of these leaves exhibited strong (resp. weak) non-Lambertian behavior, e.g., bay laurel and holly (resp. bamboo). Also, some of them had regular (resp. non-regular) surfaces, e.g., bay laurel (resp. ivy and holly). All of these leaves were harvested in March, 2015 and selected on a color basis, ranging from dark green for leaves with a high chlorophyll content, to yellow for senescent leaves. As illustrated in Fig. 2, each leaf was placed on a translation stage and was imaged in three positions using a $20^{\circ}$ tilted stand. As a result, the average incident angles $\bar{\theta}_{i}$ were $0^{\circ}, 20^{\circ}$ and $40^{\circ}$ for VNIR camera, and $10^{\circ}, 30^{\circ}$ and $50^{\circ}$ for SWIR camera. Note that the translation stage had low reflectivity so we assumed that, after being transmitted through the leaf, the light reflected from the translation stage 
and re-transmitted through the leaf was negligible.

In addition, a SWIR image of a sugar beet leaf (Beta vulgaris) was acquired, especially because this species shows interesting features in this spectral range, i.e., very high equivalent water thickness $\left(C_{w}>0.02 \mathrm{~cm}\right)$ and low leaf mass per area $\left(C_{m}<0.005\right.$ g.cm $\left.{ }^{-2}\right)$, as well as a strongly non-Lambertian and non-regular surface, thereby illustrating well the relevance of our model. This leaf was only imaged in horizontal position, its surface being already highly non-regular.

\subsubsection{Reference measurements}

For each leaf, after spectral measurements, several leaf disks (from two to seven disks depending on leaf size) of known area were sampled using a cork borer. Each disk position was then recorded for further reference. A Dualex scientific $+^{\mathrm{TM}}$ (Force-A, Orsay, France), hereafter called Dualex, was used to measure the chlorophyll $\mathrm{a}+\mathrm{b}$ content $C_{a b}$ in every disk. This leafclip allows non-destructive transmittance-based $C_{a b}$ measurements characterized by an accuracy of around $5 \mu \mathrm{g} . \mathrm{cm}^{-2}$ (Cerovic et al., 2012). Compared with the well known SPAD-502 ${ }^{\mathrm{TM}}$ (Minolta, Japan), the Dualex uses a higher $C_{a b^{-}}$-sensitive wavelength $(710 \mathrm{~nm})$ that allows it to have a nearly linear response to variation in $C_{a b}$ for $C_{a b}<40 \mu \mathrm{g} . \mathrm{cm}^{-2}$. However, for dicotyledons, saturation occurring beyond $40 \mu \mathrm{g} . \mathrm{cm}^{-2}$ leads the Dualex to underestimate such $C_{a b}$ values (Cerovic et al., 2012). The higher $C_{a b}$, the greater the underestimation.

Leaf disks were then weighted and placed in a drying oven at $75^{\circ} \mathrm{C}$ for $48 \mathrm{~h}$. Subsequently, their dry mass was measured to obtain the equivalent water 
Table 2: Characteristics of reference measurements for the main data set.

\begin{tabular}{ccccc}
\hline Parameter & Number of samples & Mean & Min/Max & Standard deviation \\
\hline$C_{a b}\left[\mu \mathrm{g} \cdot \mathrm{cm}^{-2}\right]$ & 93 & 29.4 & $5.2 / 54.6$ & 11.1 \\
$C_{w}[\mathrm{~cm}]$ & 22 & 0.0104 & $0.0049 / 0.0164$ & 0.0040 \\
$C_{m}\left[\mathrm{~g} \cdot \mathrm{cm}^{-2}\right]$ & 22 & 0.0091 & $0.0052 / 0.0129$ & 0.0023 \\
\hline
\end{tabular}

thickness $C_{w}$ (in $\left.[\mathrm{cm}]\right)$ and leaf mass per area $C_{m}$ (in $\left.\left[\mathrm{g} . \mathrm{cm}^{-2}\right]\right)$ as follows:

$$
\begin{aligned}
C_{w} & =\frac{F W-D W}{A} \times d_{w} \\
C_{m} & =\frac{D W}{A}
\end{aligned}
$$

where $F W$ and $D W$ are the fresh and dry weights of leaf disks respectively, $A$ is the total disk area, and $d_{w}=1 \mathrm{~g} . \mathrm{cm}^{-3}$ is the water density.

Therefore, for each leaf, several measurements of $C_{a b}$ and one measurement of $C_{w}$ and $C_{m}$ were available. The ranges of $C_{a b}, C_{w}$ and $C_{m}$ for the main data set are reported in Table 2 and were similar to those found in the literature (Féret et al., 2008; Danson \& Bowyer, 2004).

\subsection{Retrieval of leaf parameters from model inversion}

In this study, we used the PROSPECT (Leaf Optical Properties Spectra) model to simulate the leaf DHR from 400 to $2500 \mathrm{~nm}$ as a function of the leaf biochemistry and structure. The original version developed by Jacquemoud \& Baret (1990) has been successively improved over the years to take into account other biochemical components and a broader spectral range (Jacquemoud et al., 1996; Baret \& Fourty, 1997a; Jacquemoud et al., 2000; Féret et al., 2008; Gerber et al., 2011). The considered PROSPECT 5b version was developed by Féret et al. (2008) (available at http://teledetection.ipgp. 
jussieu.fr/prosail/) and simulates the leaf DHR as a function of the leaf structure parameter $N$, chlorophyll a+b content $C_{a b}$, carotenoid content $C_{c x}$, brown pigment content $C_{b p}$, equivalent water thickness $C_{w}$ and leaf mass per area $C_{m}$.

In the following, leaf parameters were retrieved based on model inversion. The parameters that were only affecting the reflected radiation in the VNIR range (resp. the SWIR range), were kept constant when considering the SWIR range (resp. the VNIR range) so as to reduce the estimation uncertainty. Therefore, because water absorption is weak in the VNIR range and only occurs around $970 \mathrm{~nm}$ (Curran, 1989), the VNIR range was reduced to $410-900 \mathrm{~nm}$ (i.e., 135 bands) and $C_{w}$ was fixed to the default value $0.01 \mathrm{~cm}$. The vector of input PROSPECT parameters was thus $\boldsymbol{\vartheta}_{d h r}=$ $\left[N, C_{a b}, C_{c x}, C_{b p}, C_{m}\right]^{t}$. Similarly, foliar pigments do not affect the SWIR reflectance so $C_{a b}, C_{c x}$ and $C_{b p}$ were fixed to the default values $30 \mu \mathrm{g} . \mathrm{cm}^{-2}$, $10 \mu \mathrm{g} \cdot \mathrm{cm}^{-2}$ and 0 respectively. In the SWIR range, the vector of input PROSPECT parameters was $\boldsymbol{\vartheta}_{d h r}=\left[N, C_{w}, C_{m}\right]^{t}$.

For each pixel, the combined pseudo-BRF based PROSPECT+COSINE model (named PROCOSINE hereafter) was numerically inverted by optimizing the following least square merit function:

$$
\widehat{\boldsymbol{\vartheta}}_{\text {pseudo-brf }}=\underset{\boldsymbol{\vartheta}}{\operatorname{argmin}} \sum_{\lambda_{i}}\left[R_{h s i}\left(\lambda_{i}\right)-\widetilde{R_{h s i}}\left(\lambda_{i} ; \boldsymbol{\vartheta}\right)\right]^{2}
$$

where $\boldsymbol{\vartheta}=\left[\boldsymbol{\vartheta}_{d h r}, \theta_{i}, b_{\text {spec }}\right]^{t}, R_{h s i}$ is the measured pseudo-BRF retrieved using Eq. 9 and $\widetilde{R_{h s i}}$ is the pseudo-BRF based PROCOSINE model given by Eq. 20. Similarly, the radiance-based PROCOSINE model was inverted by solving 
Table 3: Lower bounds, upper bounds and initial values of the optimization problem.

\begin{tabular}{cccc}
\hline Parameter & Lower bound & Upper bound & Initial value \\
\hline$N$ & 1 & 3.5 & 1.5 \\
$C_{a b}\left[\mu \mathrm{g} \cdot \mathrm{cm}^{-2}\right]$ & 0 & 100 & 50 \\
$C_{c x}\left[\mu \mathrm{g} \cdot \mathrm{cm}^{-2}\right]$ & 0 & 30 & 10 \\
$C_{b p}$ & 0 & 5 & 0 \\
$C_{w}[\mathrm{~cm}]$ & 0.00005 & 0.1 & 0.01 \\
$C_{m}\left[\mathrm{~g} \cdot \mathrm{cm}{ }^{-2}\right]$ & 0.001 & 0.03 & 0.01 \\
$\theta_{i}\left[{ }^{\circ}\right]$ & 0 & 90 & 20 \\
$b_{\text {spec }}$ & -0.2 & 0.6 & 0 \\
\hline
\end{tabular}

$\left(\frac{10}{20}\right.$

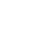

365

the following optimization problem:

$$
\widehat{\boldsymbol{\vartheta}}_{\text {radiance }}=\underset{\boldsymbol{\vartheta}}{\operatorname{argmin}} \sum_{\lambda_{i}}\left[L_{r}\left(\lambda_{i}\right)-\widetilde{L_{r}}\left(\lambda_{i} ; \boldsymbol{\vartheta}\right)\right]^{2}
$$

where $L_{r}$ is the measured spectral radiance and $\widetilde{L_{r}}$ is the radiance-based PROCOSINE model given by Eq. 21.

To reduce the number of solutions to the inverse problem, the estimation range was restricted using lower and upper bounds. These bounds as well as initial values are provided in Table 3 . Note that $C_{b p}$ and $C_{w}$ upper bounds are higher than those found in the literature because, at the sub-millimeter level, $C_{b p}$ and $C_{w}$ can be very high in necrotic regions and veins respectively. Optimization of Eq. 24 and Eq. 25 was performed using the trust-region reflective algorithm implemented in $\mathrm{MATLAB}^{\circledR}$ (version 8.0.0, The MathWorks Inc., Natick, MA, 2012) within the "lsqcurvefit" function. 


\subsection{PROCOSINE sensitivity analysis}

Before assessing PROCOSINE in terms of estimation results, we performed a global sensitivity analysis in order to study the relative contribution of each parameter to the modeled pseudo-BRF as a function of wavelength. Global sensitivity analysis informs us on which variation in the model output is produced by variation in the model input parameters, both individually and collectively through their interactions with each other. When studied as a function of wavelength, it provides interesting insights regarding the optimal spectral bands that can be used to retrieve model parameters.

In this paper, we implemented the EFAST (Extended Fourier Amplitude Sensitivity Transform) method (Saltelli et al., 1999) that was already applied to PROSAIL and PROGEOSAIL models by Bowyer \& Danson (2004). EFAST is a quantitative variance-based method, i.e., it allows the derivation of sensitivity indices from the decomposition of the total variance of the model output into variance terms induced by every input parameter. In this paper, we only used the first-order index $S_{i}$ that represents the percentage of output variance explained by the $\mathrm{i}^{\text {th }}$ parameter alone. The remaining percentage of variance is explained by interactions between parameters and is given by $S_{i n t}=1-\sum_{i} S_{i}$. $S_{i}$ (and $S_{i n t}$ ) ranges from 0 to 1 , and the higher $S_{i}$, the more sensitive the model output is to the $\mathrm{i}^{\text {th }}$ parameter.

In this study, 5000 combinations of model parameters were randomly generated using appropriate probability distributions because the latter strongly affects the results of sensitivity analysis (Bowyer \& Danson, 2004; Wallach 
Table 4: Estimated parameter distributions ( $\mu$ : mean; $\sigma$ : standard deviation; $\alpha$ : shape parameter; $\beta$ : scale parameter).

\begin{tabular}{cccccc}
\hline Parameter & Distribution & $\mu$ & $\sigma$ & $\alpha$ & $\beta$ \\
\hline$N$ & Gamma & 1.52 & 0.23 & 47.50 & 0.032 \\
$C_{a b}\left[\mu \mathrm{g} \cdot \mathrm{cm}^{-2}\right]$ & Gamma & 32.8 & 18.87 & 1.99 & 16.45 \\
$C_{c x}\left[\mu \mathrm{g} \cdot \mathrm{cm}^{-2}\right]$ & Gamma & 8.51 & 3.92 & 3.83 & 2.22 \\
$C_{w}[\mathrm{~cm}]$ & Gamma & 0.0122 & 0.0061 & 6.45 & 0.0019 \\
$C_{m}\left[\mathrm{~g} \cdot \mathrm{cm}^{-2}\right]$ & Gamma & 0.0078 & 0.0036 & 4.20 & 0.0018 \\
$\theta_{i}\left[{ }^{\circ}\right]$ & Gamma & 25 & 10 & 6.25 & 4 \\
$b_{\text {spec }}(\mathrm{VNIR})$ & Normal & -0.009 & 0.0375 & - & - \\
$b_{\text {spec }}(\mathrm{SWIR})$ & Normal & 0.0346 & 0.0403 & - & - \\
\hline
\end{tabular}

et al., 2014). The distributions of PROSPECT parameters were estimated from seventeen independent data sets made available by Féret et al. (2011); Féret, personal communication. They include a wide range of leaf spectral, chemical, and structural properties, i.e., 1417 leaves corresponding to about 120 different species from various growing conditions and developmental stages. Note that $C_{a b}, C_{c x}, C_{w}$ and $C_{m}$ data were obtained in a destructive way, whereas $N$ data were obtained from PROSPECT inversion on DHR data. It can be shown that these data are well described by Gamma distributions $(p<0.001)$. Using such distributions (instead of normal ones) prevented us from generating samples with negative content values. Regarding brown pigments, we assumed that leaves under study were green so $C_{b p}$ was set to zero. Finally, $\theta_{i}$ and $b_{\text {spec }}$ distributions were retrieved from the estimation results presented in Section 4 using a Gamma distribution for $\theta_{i}$ and a normal distribution for $b_{\text {spec }}$ (not shown). The characteristics of all of these distributions are presented in Table 4, where the shape parameter $\alpha$ 
and the scale parameter $\beta$ of the Gamma distribution were given by their maximum likelihood estimates. Note that two $b_{\text {spec }}$ distributions were used for the two spectral domains. Importantly, the covariance between parameters could not be considered when generating the samples. Consequently, the actual influences of $C_{a b}$ and $C_{c x}$ are likely to be slightly different, both parameters being highly correlated (Féret et al., 2008).

For each EFAST run, 5000 leaf reflectance spectra were therefore simulated using PROSPECT and PROCOSINE (Eq. 20) so as to compute first-order indices and interactions. Average indices were finally computed over 15 runs.

\section{Results and discussion}

\subsection{Sensitivity analysis}

In Figure 3, we compare the first-order indices and interactions obtained with PROSPECT and PROCOSINE over the VNIR and SWIR ranges. Fig. 3.a and Fig. 3.b reveal the same features already observed in previous studies using other methods and/or models (Jacquemoud \& Baret, 1990; Jacquemoud et al., 2009; Bacour et al., 2001, 2002; Bowyer \& Danson, 2004). In the visible range $(400-730 \mathrm{~nm})$, photosynthetic pigments (i.e., chlorophyll and carotenoids) drive most of the reflectance variability. Between 400 and $525 \mathrm{~nm}$ where the carotenoid absorption is the strongest (Féret et al., 2008), the contributions of $C_{a b}$ and $C_{c x}$ are similar in magnitude and range from 20 to $40 \%$. Between 525 and $730 \mathrm{~nm}$, reflectance mainly depends on $C_{a b}$, its contribution ranging from 40 to $90 \%$. The influence of interactions is higher in strong absorption regions, which means that the effects of other parameters depend on $C_{a b}$ and $C_{c x}$ values. For example, the relative increase in 
(a)

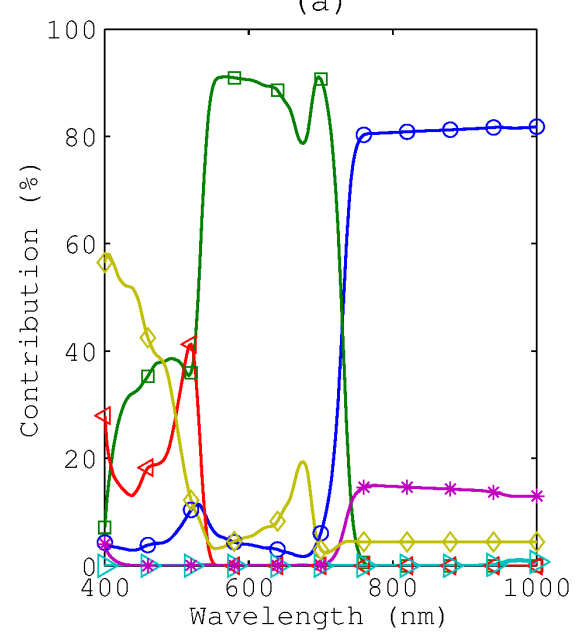

(c)

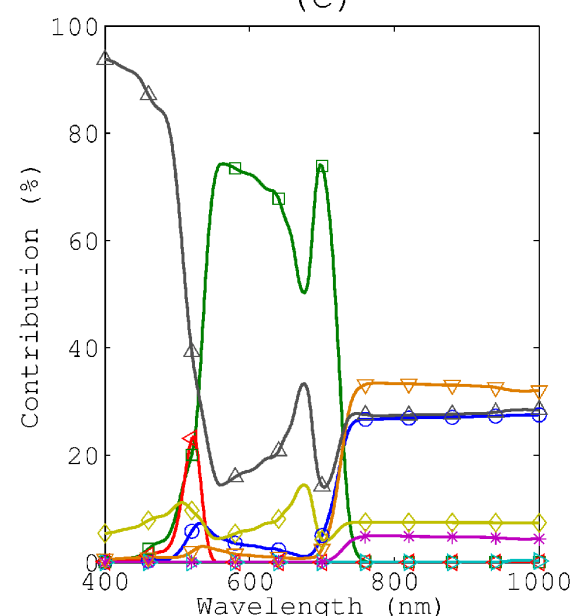

$\smile \mathrm{N} \longrightarrow \mathrm{C}_{\mathrm{ab}} \smile \mathrm{C}_{\mathrm{CX}} \longrightarrow \mathrm{C}_{\mathrm{w}}$ (b)

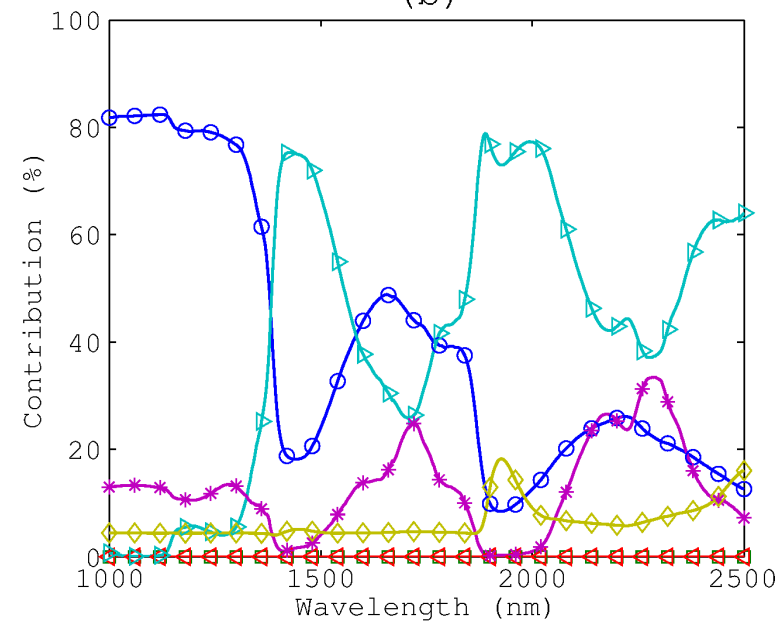

(d)

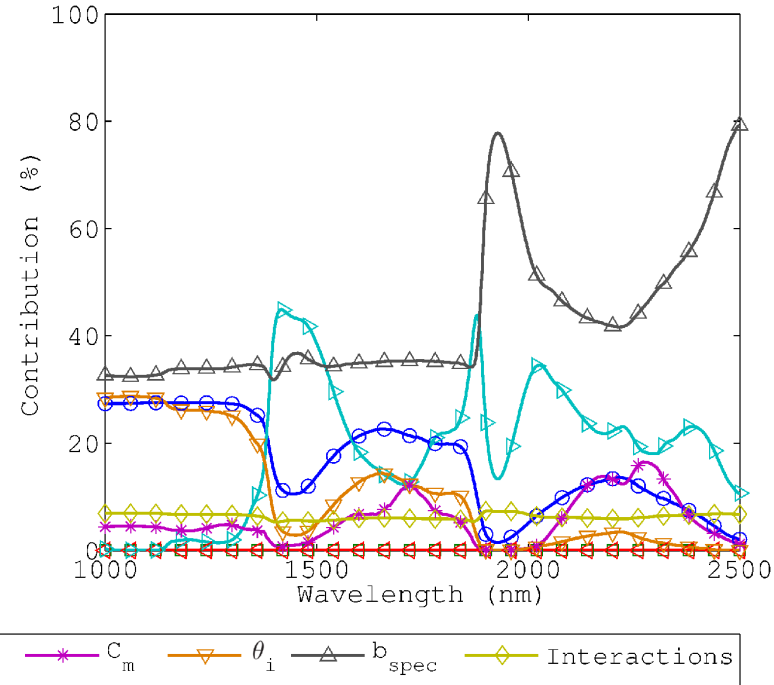

Figure 3: First-order sensitivity indices and interactions for (a) PROSPECT in the VNIR range, (b) PROSPECT in the SWIR range, (c) PROCOSINE in the VNIR range, and (d) PROCOSINE in the SWIR range $\left(\theta_{s}=26^{\circ}\right)$. 
reflectance due an increase in $N$ (reflecting higher scattering within the leaf internal structure) will be high in case of low absorption and low in case of strong absorption.

In the NIR plateau, variation in reflectance is mainly produced by variation in leaf structure (80\%) and leaf mass per area (14\%) through scattering processes (Knyazikhin et al., 2013). In the SWIR range, only leaf structure, equivalent water thickness and leaf mass per area have significant contributions. In particular, $C_{w}$ strongly influences reflectance in the water absorption peaks located around 1450 and $1950 \mathrm{~nm}$ (about 75\%), whereas variation in $C_{m}$ has a significant contribution around 1720 and $2250 \mathrm{~nm}$ (resp. $24 \%$ and $30 \%)$.

Taking into account BRDF effects and leaf orientation through the $b_{\text {spec }}$ and $\theta_{i}$ parameters within the PROCOSINE model modifies the reflectance sensitivity as shown in Figure 3.c and Figure 3.d. Compared with PROSPECT sensitivity analysis, the overall contributions of PROSPECT parameters are mostly similar in shape but still, they decrease to account for variance in $b_{\text {spec }}$ and $\theta_{i}$. For example, the $C_{a b}$ contribution at around $550 \mathrm{~nm}$ decreases from 90 to $70 \%$.

The contributions of $b_{\text {spec }}$ and $\theta_{i}$ are quite different. Overall, variance in $b_{\text {spec }}$ highly influences reflectance in strong absorption regions (both due to $C_{a b}$ and $C_{w}$ ), accounting for up to $90 \%$ near $400 \mathrm{~nm}$ and $75 \%$ near 1930 and $2500 \mathrm{~nm}$. In these regions, the diffuse part of reflectance is very low so the influence of the surface-reflected flux (that does not interact with the leaf volume) dominates. Interestingly, an analogy can be made with high- 
resolution water remote sensing, in that the sun glint (i.e., the amount of flux directly reflected at the water surface) is usually estimated in the NIR region, where the water-leaving signal is negligible because of strong water absorption (Hochberg et al., 2003). Overall, the $b_{\text {spec }}$ contribution is always greater than $15 \%$ between 400 and $2500 \mathrm{~nm}$, thereby indicating that this parameter should not be neglected when dealing with close-range hyperspectral images.

The contribution of the incident angle $\theta_{i}$ significantly varies over the whole spectral domain. It mostly affects reflectance in the NIR plateau, accounting for about $30 \%$ of the variance of the PROCOSINE output. Conversely, its influence is much weaker in the visible range, where its contribution does not exceed $5 \%$, as well as in water absorption regions. Generally, because it affects the model through a multiplicative term (see Eq. 20 and Eq. 21), the spectral profile of its contribution is similar to a common leaf spectral signature: the higher the reflectance, the higher its contribution. Interestingly, this profile is also similar to the contribution of the average leaf angle (ALA) to the PROSAIL model (Jacquemoud et al., 2009). In the latter case, ALA has more influence at weakly absorbing wavelengths because the effect of multiple scattering within the canopy is stronger (Knyazikhin et al., 2013).

To summarize, this sensitivity analysis shows that both $b_{\text {spec }}$ and $\theta_{i}$ significantly affect the measured signal and definitively have to be taken into account in the case of close-range imaging spectroscopy. 


\subsection{Quantitative assessment of model inversion}

The performances of model inversion were quantitatively assessed both in terms of spectral fitting and parameter retrieval. Note that we had only reference measurements integrated over one (for $C_{a b}$ ) or several (for $C_{w}$ and $\left.C_{m}\right)$ leaf $\operatorname{disk}(\mathrm{s})$ of a few square centimeters. As a result, in this section, the means and standard deviations of $C_{a b}, C_{w}$ and $C_{m}$ estimated values were computed from the values estimated within the corresponding leaf disk(s).

\subsubsection{PROCOSINE spectral accuracy}

We first quantified how accurate was PROCOSINE in fitting the pseudo$\mathrm{BRF}$ and radiance measurements retrieved at the pixel level. In Fig. 4, we show some fitting results obtained with an ivy leaf both for the VNIR and SWIR ranges, this leaf surface being non-Lambertian and non-regular.

Overall, strong agreements were obtained between measured and simulated spectra in the two ranges (RMSE $<4 \%)$. As expected, high $b_{\text {spec }}$ values were retrieved in areas affected by specular reflection, and high $\theta_{i}$ values were retrieved where the light incident angle was high. Compared with the VNIR range, the obtained RMSE were about twice higher for the SWIR range. This is in agreement with the mismodeling of specular reflection in the SWIR range mentioned in Section 2.2.1 and discussed further in Section 4.2.4. These results thus demonstrate the accuracy of PROCOSINE in accounting for the spectral variability induced by BRDF effects and leaf orientation, especially in the VNIR range. 
(a)

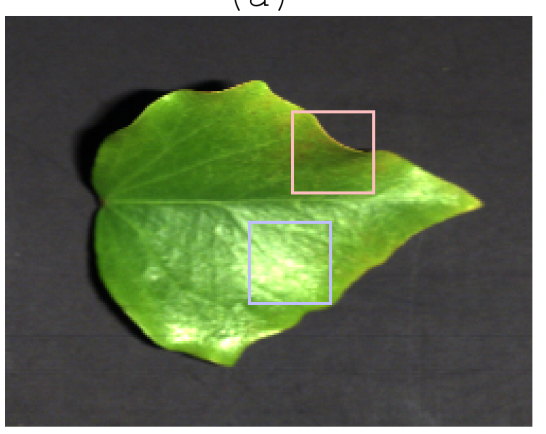

(b)
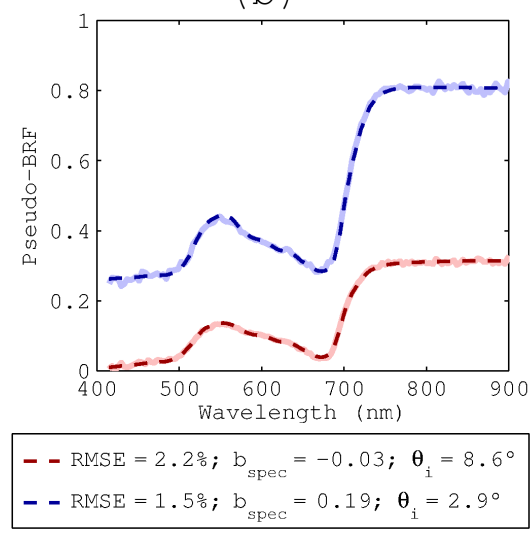

( c)

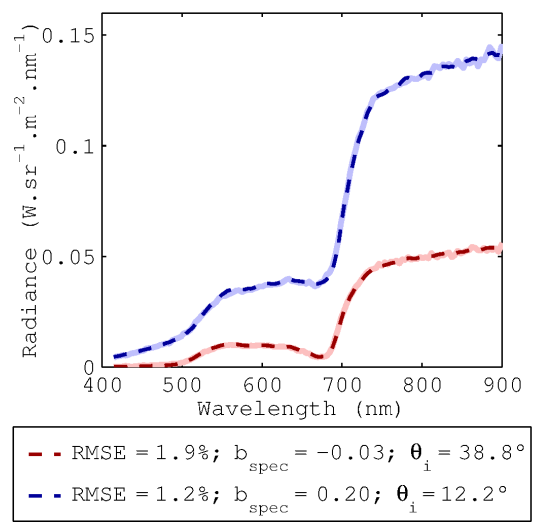

(d)

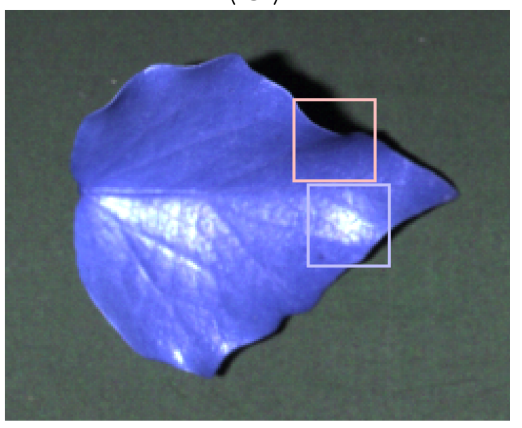

(e)
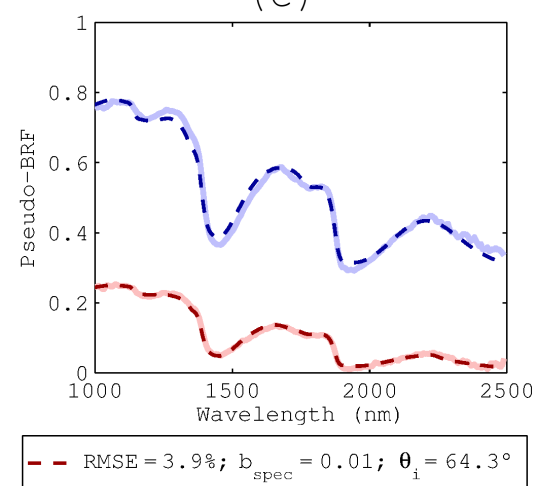

- $\mathrm{RMSE}=3.6 \% ; \mathrm{b}=0.39 ; \theta_{i}=50.0^{\circ}$

( f)

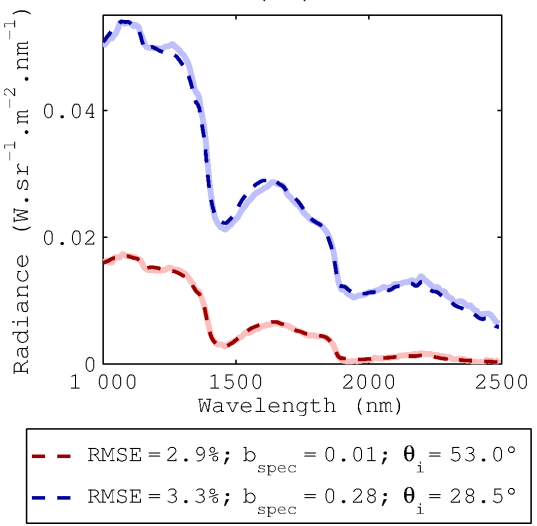

Figure 4: Fitting results obtained for the VNIR range (a-c) and the SWIR range (d-f) using the pseudo-BRF model (b,e) and the radiance model (c,f). Solid (resp. dashed) lines correspond to measurements (resp. simulations). Blue curves correspond to areas strongly affected by specular reflection, while red curves correspond to areas characterized by a high incident angle. 

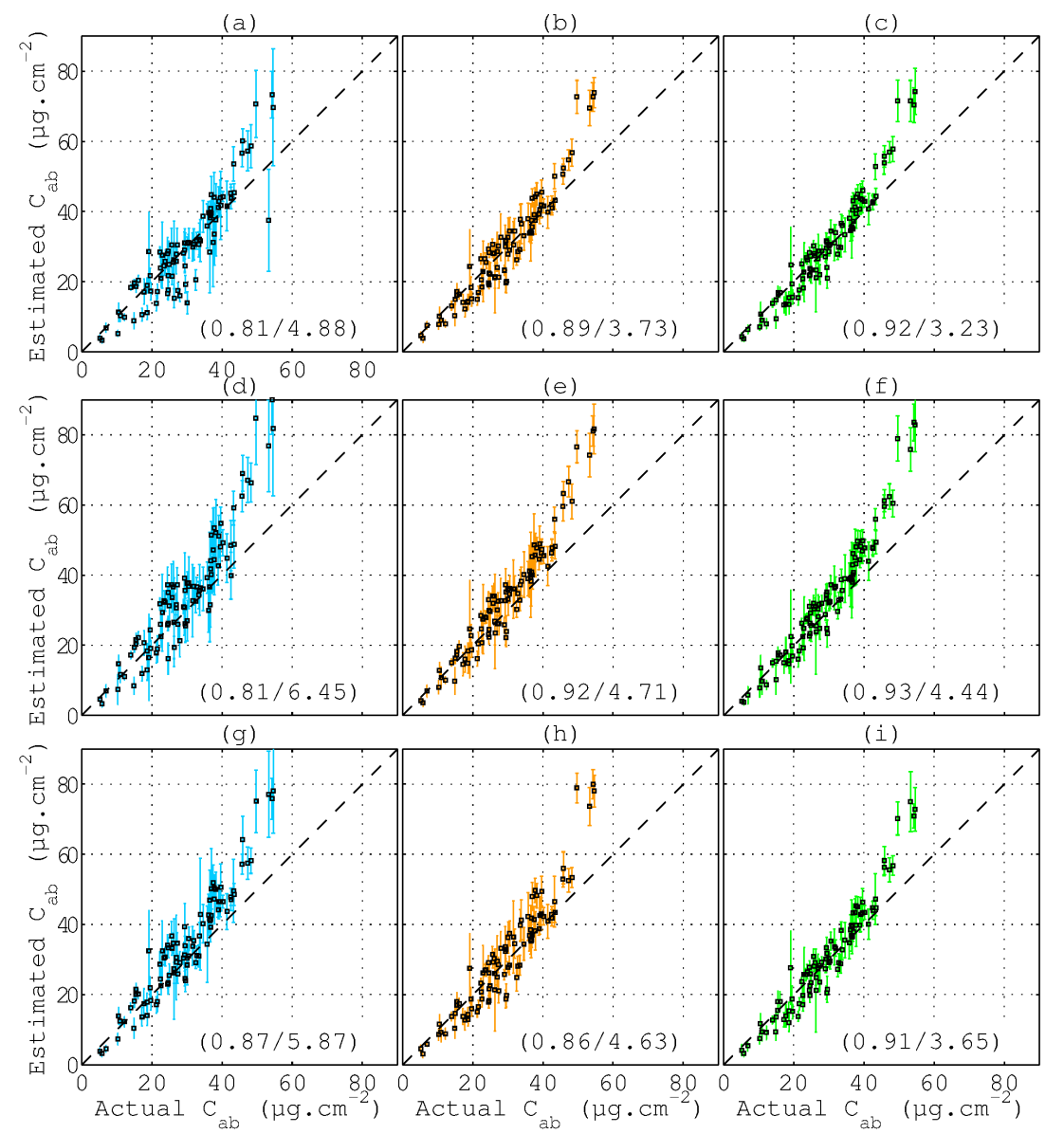

Figure 5: $C_{a b}$ estimation results (mean +/- standard deviation) obtained with PROSPECT (blue), the pseudo-BRF based PROCOSINE model (orange), and the radiance-based PROCOSINE model (green) and with $\bar{\theta}_{i}=20^{\circ}$ (a-c), $\bar{\theta}_{i}=0^{\circ}$ (d-f) and $\bar{\theta}_{i}=40^{\circ}$ (g-i). The $\mathrm{R}^{2}$ and RMSE values are computed for actual $C_{a b}$ values lower than $40 \mu \mathrm{g} . \mathrm{cm}^{-2}$ and are given in parentheses. 


\subsubsection{Results of parameter retrievals in the VNIR range}

As recalled by the sensitivity analysis, the VNIR reflectance is mainly driven by the chlorophyll $\mathrm{a}+\mathrm{b}$ content. In Fig. 5, we show the means and standard deviations of $C_{a b}$ estimation obtained for each leaf disk with leaves in horizontal position (Figure 5.a-c) and tilted position (Figure 5.d-i). We compare the results obtained with PROSPECT (in blue), the pseudo-BRF based PROCOSINE model (in orange) and the radiance-based PROCOSINE model (in green). Because of the saturation in the reference measurement (see Section 3.1.2), the RMSE and $\mathrm{R}^{2}$ values were computed from samples for which the measured $C_{a b}$ values were lower than $40 \mu \mathrm{g} . \mathrm{cm}^{-2}$.

On average, when tested with horizontal leaves, the radiance-based PROCOSINE model led to a higher accuracy $\left(\mathrm{R}^{2}=0.92 ; \mathrm{RMSE}=3.23 \mu \mathrm{g} . \mathrm{cm}^{-2}\right)$ than the pseudo-BRF PROCOSINE model $\left(\mathrm{R}^{2}=0.89 ; \mathrm{RMSE}=3.73 \mu \mathrm{g} . \mathrm{cm}^{-2}\right)$ and PROSPECT $\left(\mathrm{R}^{2}=0.81 ; \mathrm{RMSE}=4.88 \mu \mathrm{g} \cdot \mathrm{cm}^{-2}\right)$. Applying the same models to leaves in tilted position decreased the estimation accuracy, however to a variable extent. For $\bar{\theta}_{i}=0^{\circ}$, the radiance-based PROCOSINE model $\left(\mathrm{R}^{2}=0.93 ; \mathrm{RMSE}=4.44 \mu \mathrm{g} . \mathrm{cm}^{-2}\right)$ still performed better than the pseudo-BRF based PROCOSINE model $\left(\mathrm{R}^{2}=0.92 ; \mathrm{RMSE}=4.71 \mu \mathrm{g} \cdot \mathrm{cm}^{-2}\right)$ and PROSPECT $\left(\mathrm{R}^{2}=0.81 ; \mathrm{RMSE}=6.45 \mu \mathrm{g} \cdot \mathrm{cm}^{-2}\right)$. The same trend was also observed for $\bar{\theta}_{i}=40^{\circ}$, the radiance-based PROCOSINE model leading to a better accuracy $\left(\mathrm{R}^{2}=0.91 ; \mathrm{RMSE}=3.65 \mu \mathrm{g} . \mathrm{cm}^{-2}\right)$ than the pseudoBRF based PROCOSINE model $\left(\mathrm{R}^{2}=0.86 ; \mathrm{RMSE}=4.63 \mu \mathrm{g} . \mathrm{cm}^{-2}\right)$ and PROSPECT $\left(\mathrm{R}^{2}=0.87\right.$;MSE $\left.=5.87 \mu \mathrm{g} \cdot \mathrm{cm}^{-2}\right)$.

Overall, both PROCOSINE implementations obtained significantly lower 

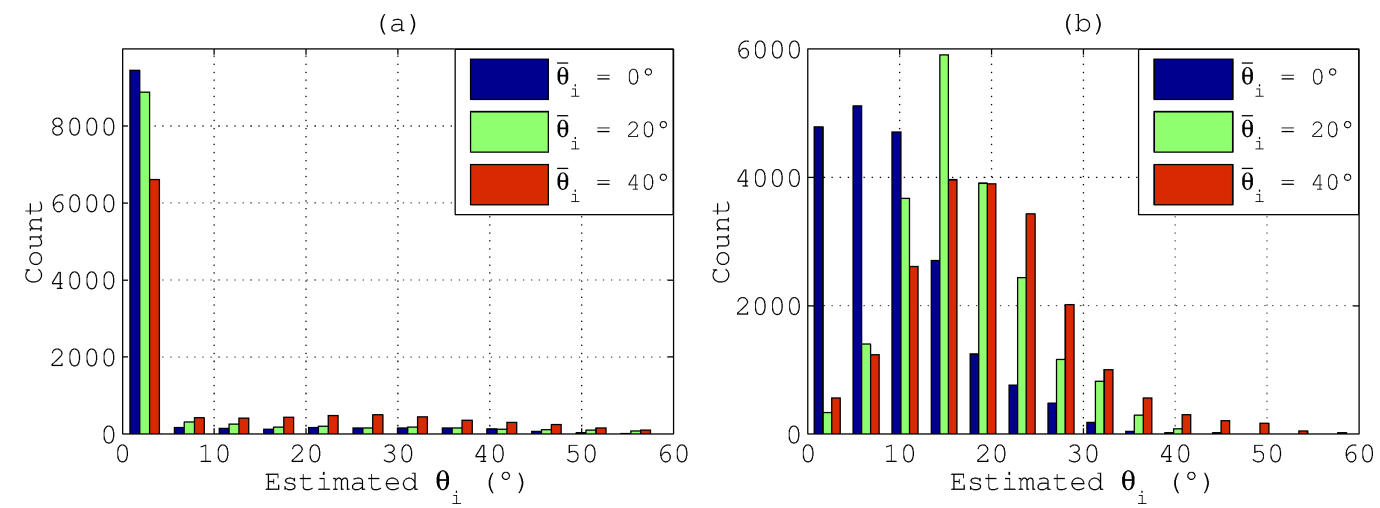

Figure 6: Histograms of estimated $\theta_{i}$ values for every pixel for the VNIR range and the three acquisition configurations $\left(\bar{\theta}_{i}=0^{\circ}, \bar{\theta}_{i}=20^{\circ}\right.$, and $\bar{\theta}_{i}=40^{\circ}$ ): (a) pseudo-BRF based PROCOSINE model, and (b) radiance-based PROCOSINE model.

standard deviations of $C_{a b}$ estimation than PROSPECT.

Lastly, for $C_{a b}>40 \mu \mathrm{g} . \mathrm{cm}^{-2}$, model inversion always provided higher $C_{a b}$ values than those measured with the Dualex.

The incident angle estimation within the whole leaves is evaluated in Fig. 6. The results are presented using histograms to account for within-leaf variability of leaf orientation. For both model implementations, we represent the distributions of $\theta_{i}$ values estimated for every pixel for the three acquisition configurations (i.e., average incident angles $\bar{\theta}_{i}$ of $0^{\circ}, 20^{\circ}$, and $40^{\circ}$ ).

Surprisingly, strong differences were observed between both model inversions. On the one hand, the pseudo-BRF based model gave poor estimation results with estimated $\bar{\theta}_{i}$ of $3.3^{\circ}, 5.1^{\circ}$ and $11.7^{\circ}$ (and the same estimated distribution mode, i.e., $2.4^{\circ}$ ) for $\bar{\theta}_{i}=0^{\circ}, \bar{\theta}_{i}=20^{\circ}$, and $\bar{\theta}_{i}=40^{\circ}$ respectively. On the other hand, the radiance-based model gave better estimation results with estimated $\bar{\theta}_{i}$ of $9.7^{\circ}, 16.9^{\circ}$ and $19.6^{\circ}$ (and estimated distribution modes of $6.3^{\circ}, 14.8^{\circ}$ 
and $14.8^{\circ}$ ) for $\bar{\theta}_{i}=0^{\circ}, \bar{\theta}_{i}=20^{\circ}$, and $\bar{\theta}_{i}=40^{\circ}$ respectively.

It is worth noting that poorer angle estimation results were generally obtained for bamboo leaves (results not shown) with estimated distribution modes of $15.4^{\circ}, 28.6^{\circ}$ and $28.6^{\circ}$ ) for $\bar{\theta}_{i}=0^{\circ}, \bar{\theta}_{i}=20^{\circ}$, and $\bar{\theta}_{i}=40^{\circ}$ respectively.

\subsubsection{Results of parameter retrievals in the SWIR range}

Regarding PROSPECT original parameters, variation in SWIR reflectance is mainly produced by variation in equivalent water thickness, leaf mass per area and leaf structure.

In Fig. 7, we show the means and standard deviations of $C_{w}$ estimation obtained with leaves in horizontal position (Fig. 7.a-c) and in tilted position (Fig. 7.d-i). For horizontal leaves, PROCOSINE performed better than PROSPECT in terms of $\mathrm{R}^{2}\left(\mathrm{R}^{2}=0.91\right.$ for PROSPECT, $\mathrm{R}^{2}=0.93$ for the pseudo-BRF based PROCOSINE model and $\mathrm{R}^{2}=0.94$ for the radiancebased PROCOSINE model), but worse in terms of RMSE $(\mathrm{RMSE}=0.0016 \mathrm{~cm}$, $\mathrm{RMSE}=0.0083 \mathrm{~cm}$ and $\mathrm{RMSE}=0.0025 \mathrm{~cm}$ resp.). However, the PROCOSINE performances remained mostly unchanged when applied to tilted leaves, while PROSPECT obtained poorer performances, either for $\bar{\theta}_{i}=10^{\circ}$ (a $125 \%$ increase in RMSE) or $\bar{\theta}_{i}=50^{\circ}$ (a $15 \%$ decrease in $\mathrm{R}^{2}$ ).

The RMSE values obtained with PROCOSINE were primarily due to a systematic bias, the $\mathrm{R}^{2}$ values being higher than 0.91 for the three acquisition configurations. This bias was more pronounced for the pseudo-BRF based model, and led to a systematic overestimation of $C_{w}$.

Lastly, similarly to $C_{a b}$ estimation, PROCOSINE obtained lower standard deviations of $C_{w}$ estimation than PROSPECT, especially for tilted leaves. 

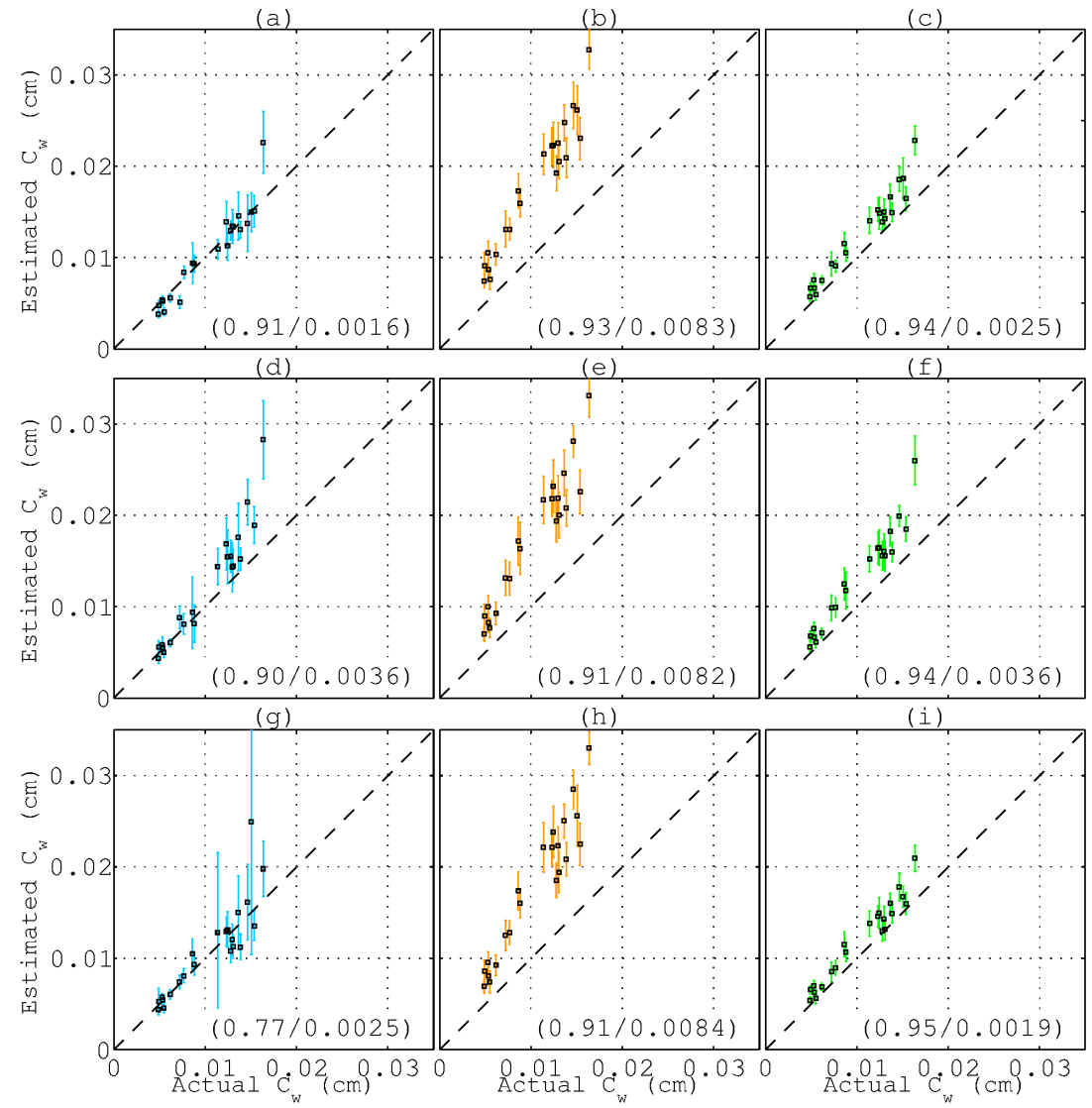

Figure 7: $C_{w}$ estimation results (mean $+/$ - standard deviation) obtained with PROSPECT (blue), the pseudo-BRF based PROCOSINE model (orange), and the radiance-based PROCOSINE model (green) and with $\bar{\theta}_{i}=30^{\circ}$ (a-c), $\bar{\theta}_{i}=10^{\circ}$ (d-f) and $\bar{\theta}_{i}=50^{\circ}$ (g-i). $\mathrm{R}^{2}$ and RMSE values are given in parentheses. 

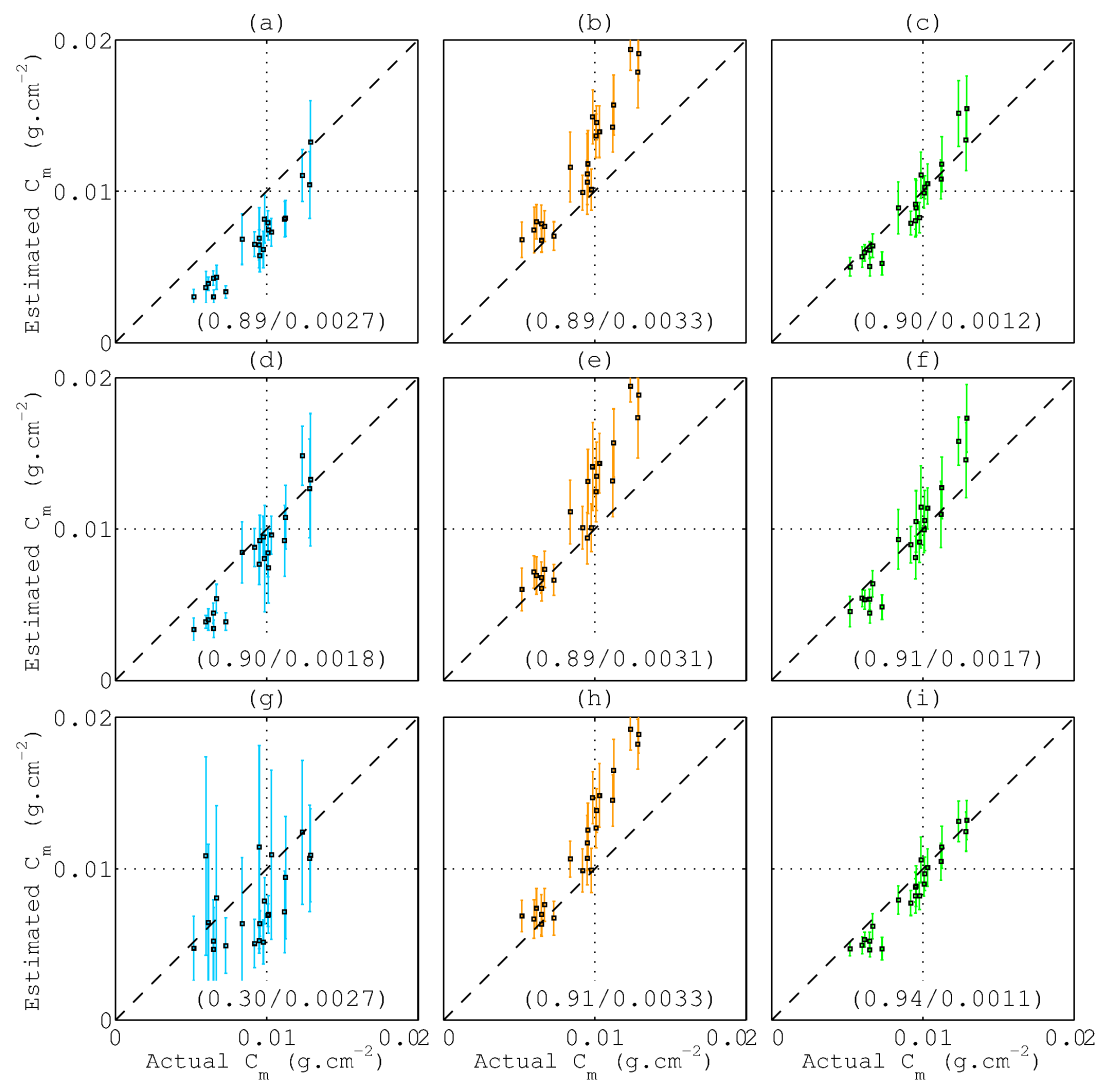

Figure 8: $C_{m}$ estimation results (mean $+/$ - standard deviation) obtained with PROSPECT (blue), the pseudo-BRF based PROCOSINE model (orange), and the radiance-based PROCOSINE model (green) and with $\bar{\theta}_{i}=30^{\circ}(\mathrm{a}-\mathrm{c}), \bar{\theta}_{i}=10^{\circ}(\mathrm{d}-\mathrm{f})$ and $\bar{\theta}_{i}=50^{\circ}(\mathrm{g}-\mathrm{i}) . \mathrm{R}^{2}$ and RMSE values are given in parentheses. 
(a)

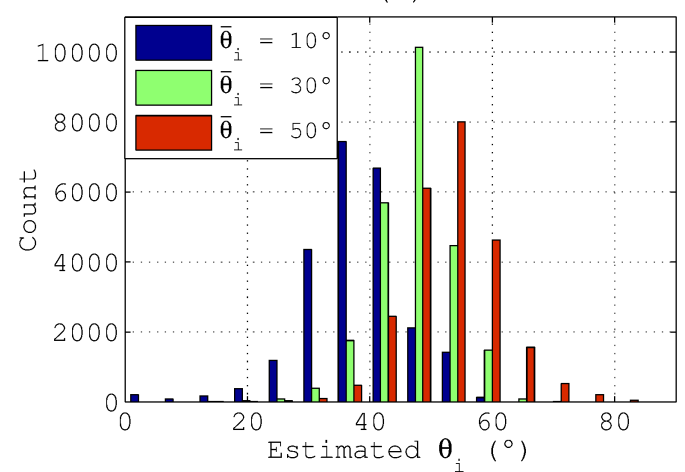

(b)

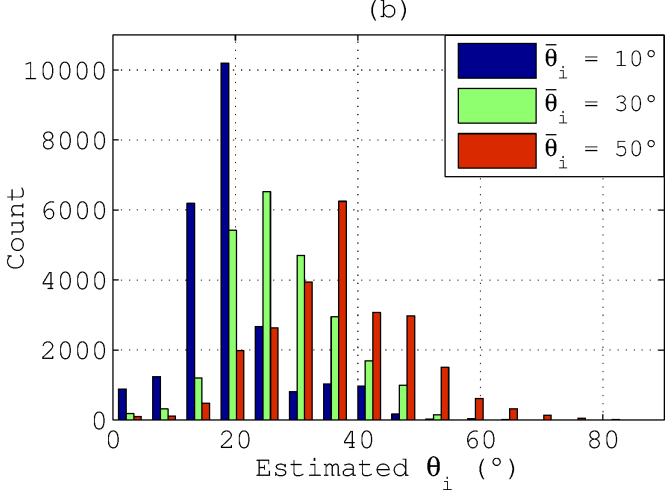

Figure 9: Histograms of estimated $\theta_{i}$ values in every pixel for the SWIR range and the three acquisition configurations $\left(\bar{\theta}_{i}=10^{\circ}, \bar{\theta}_{i}=30^{\circ}\right.$, and $\bar{\theta}_{i}=50^{\circ}$ ): (a) pseudo-BRF based PROCOSINE model, and (b) radiance-based PROCOSINE model.

The $C_{m}$ retrieval results are presented in Fig. 8 in a similar fashion to $C_{w}$. The radiance-based PROCOSINE model gave significantly lower RMSE than the pseudo-BRF based PROCOSINE model and PROSPECT, i.e., RMSE = $0.0013 \mathrm{~g} . \mathrm{cm}^{-2}, \mathrm{RMSE}=0.0032 \mathrm{~g} . \mathrm{cm}^{-2}$, and RMSE $=0.0024 \mathrm{~g} . \mathrm{cm}^{-2}$ respectively on average for the three acquisition configurations. The PROCOSINE performances weakly depended on leaf position, whereas the $\mathrm{R}^{2}$ dropped from 0.89 for $\bar{\theta}_{i}=10^{\circ}$ and $\bar{\theta}_{i}=30^{\circ}$, to 0.30 for $\bar{\theta}_{i}=50^{\circ}$ when inverting only PROSPECT. For such high incident angles, the uncertainty of $C_{m}$ estimation was much higher with PROSPECT than with PROCOSINE.

Lastly, $C_{m}$ was generally underestimated by PROSPECT and overestimated by the pseudo-BRF based PROCOSINE model.

The two PROCOSINE inversions were also compared based on $\theta_{i}$ estimation results in Fig. 9, in which we represent the histograms of estimated $\theta_{i}$ distributions. As expected, in both cases, the highest (resp. the lowest) 
angles were mostly retrieved from the $\bar{\theta}_{i}=50^{\circ}\left(\right.$ resp. $\bar{\theta}_{i}=10^{\circ}$ ) configuration, the dispersions around the mean values being due to the variation in leaf orientation. $\theta_{i}$ was more accurately predicted using the radiance-based model since the estimated $\bar{\theta}_{i}$ were $19.7^{\circ}, 27.7^{\circ}$ and $36.4^{\circ}$ for $\bar{\theta}_{i}=10^{\circ}, \bar{\theta}_{i}=30^{\circ}$, and $\bar{\theta}_{i}=50^{\circ}$ respectively. The pseudo-BRF based model globally overestimated $\theta_{i}$ since the estimated $\bar{\theta}_{i}$ were $38.0^{\circ}, 47.2^{\circ}$ and $53.0^{\circ}$ for $\bar{\theta}_{i}=10^{\circ}, \bar{\theta}_{i}=30^{\circ}$, and $\bar{\theta}_{i}=50^{\circ}$ respectively.

Similarly to the VNIR range, poorer estimation results were generally obtained for bamboo leaves (results not shown). For example, the radiancebased model led to estimated $\bar{\theta}_{i}$ of $38.0^{\circ}, 43.4^{\circ}$ and $47.0^{\circ}$ for $\bar{\theta}_{i}=10^{\circ}, \bar{\theta}_{i}=30^{\circ}$, and $\bar{\theta}_{i}=50^{\circ}$ respectively.

\subsubsection{Discussion about parameter retrievals}

Estimation of chlorophyll content. In the VNIR range, both PROCOSINE implementations performed better than PROSPECT in retrieving $C_{a b}$. For leaves in horizontal position, the $34 \%$ improvement in RMSE when using the radiance-based PROCOSINE model instead of PROSPECT is likely to be due mainly to the modeling of BRDF effects. Not taking them into account leads to a greater uncertainty as shown through the estimation standard deviations computed within each leaf disk. For example, as illustrated in Fig. 5.a with the sample corresponding to an actual mean value of $53 \mu \mathrm{g} . \mathrm{cm}^{-2}$, a local BRDF effect induces an overall increase in reflectance, which is misinterpreted by PROSPECT as a decrease in $C_{a b}$. This result confirms one of the conclusions drawn from the sensitivity analysis, which has already showed that $b_{\text {spec }}$ significantly affects leaf close-range hyperspectral measurements.

The difference between PROCOSINE and PROSPECT was even greater 
when considering leaves in tilted position, for which the modeling of leaf inclination was more important. In particular, for the highest incident angle $\left(\bar{\theta}_{i}=40^{\circ}\right)$, both the modeling of BRDF effects and leaf orientation within the radiance-based PROCOSINE model led to an actual $38 \%$ improvement over PROSPECT. It is worth noting that taking $\theta_{i}$ into account improves the $C_{a b}$ estimation results even if $C_{a b}$ is mainly retrieved between 400 and $750 \mathrm{~nm}$, in which the effect of $\theta_{i}$ is lower (see Fig. 3.c). In fact, the difference in retrieval performance in VNIR between PROSPECT and PROCOSINE is even greater for $N$ leaf structure parameter that mainly affects reflectance in the NIR plateau, where both $\theta_{i}$ and $b_{\text {spec }}$ have a strong influence on reflectance ( $N$ maps are only shown for the SWIR range, see Fig. 12).

The PROCOSINE inversion performed well compared with the accuracy provided by the Dualex manufacturer, i.e., RMSE $=5 \mu \mathrm{g} . \mathrm{cm}^{-2}$ (Cerovic et al., 2012), especially considering that spectral measurements are affected by extra variability and that the method is based on reflectance, which is known to be less sensitive to $C_{a b}$ than transmittance (Baret \& Fourty, 1997b). Importantly, the apparent $C_{a b}$ overestimation for $C_{a b}>40 \mu \mathrm{g} \cdot \mathrm{cm}^{-2}$ was rather due to the saturation in the reference measurement (see Section 3.1.2), as also suggested by the similar trend observed for both PROSPECT and PROCOSINE. Further experiments conducted on hyperspectral data associated with laboratory extraction based measurements of pigment contents (including $C_{c x}$ ) would, however, be interesting to fully characterize the pigment retrieval.

The main difference between both PROCOSINE inversions arose for high incident angles, since the RMSE decreased by $21 \%$ when using the radiance- 
based model. This result confirms that this implementation better handles variation in leaf orientation as displayed in Fig. 6.

Estimation of equivalent water thickness and leaf mass per area. Somewhat different observations could be made from the SWIR range. In most cases, the retrievals of $C_{w}$ and $C_{m}$ resulted in lower RMSE when using the radiancebased PROCOSINE model. In particular, the $C_{m}$ estimation was very accurate even if $C_{m}$ is not the most influential parameter in this spectral domain (see Fig. 3.d). The poor $\mathrm{R}^{2}$ value and very high standard deviations obtained with PROSPECT and $\bar{\theta}_{i}=50^{\circ}$ again demonstrate the need for accounting for BRDF effects and leaf orientation, especially when the targeted parameter does not have a strong influence on reflectance.

However, as recalled in Section 2.2.1, BRDF effects are not properly taken into account for the SWIR range because, unlike the VNIR range, $b_{\text {spec }}$ depends on wavelength. Indeed, the BRDF directional component depends on the leaf refractive index (Bousquet et al., 2005), whose imaginary part is proportional to absorption (Born \& Wolf, 1980). Especially at the far end of the SWIR range, water absorption is so strong (Hale \& Querry, 1973) that the imaginary part of the leaf refractive index is no longer negligible compared with the real part (Gerber et al., 2011). This may explain why PROSPECT is more accurate than PROCOSINE in retrieving $C_{w}$ on leaves in horizontal position. Presumably, PROCOSINE systematically overestimates $C_{w}$ to mimic the decrease of the leaf refractive index (Féret et al., 2008). The compensation between $C_{w}$ and $b_{\text {spec }}$ allows the proposed models to be adjusted to the SWIR range and to obtain better estimation results 
for other PROSPECT parameters such as $C_{m}$. For leaves in tilted position, the influence of leaf orientation, whose modeling does not depend on wavelength, again makes the radiance-based PROCOSINE model more accurate than PROSPECT.

Importantly, the high $\mathrm{R}^{2}$ values obtained with PROCOSINE for $C_{w}$ and $C_{m}$ retrievals make it possible to calibrate strong linear relationships between the actual values and the estimated values. Indeed, in every case, the obtained RMSE is driven more by the estimation bias than by the variance. As carried out by Cheng et al. (2011), applying such an indirect retrieval could correct this bias and significantly enhance the estimation results obtained with a given experimental setup.

Angle estimation. Both in the VNIR and SWIR ranges, the performances of PROCOSINE weakly depended on leaf position. This tends to prove that non-regular surfaces can be handled properly by introducing the $\theta_{i}$ parameter. However, some clear differences arose between pseudo-BRF and radiance inversions. Overall, inverting the radiance-based model provided the best results, the strongest difference between both ranges lying in $\theta_{i}$ estimation, especially in the VNIR range. $\theta_{i}$ was indeed better retrieved using radiancebased inversion, the $\theta_{i}$ underestimation for high incident angles and both ranges being presumably due to the assumption $\frac{1-\rho_{\text {spec }}\left(\theta_{i} ; 2 \pi\right)}{1-\rho_{\text {spec }}(0 ; 2 \pi)} \approx 1$ in Eq. 20 . It could be shown that confounding effects between $\theta_{i}$ and $N$ (as well as $C_{m}$ in the VNIR range) were responsible for poor $\theta_{i}$ estimation results obtained through pseudo-BRF inversion. For example, as observed in Fig. 3, both $\theta_{i}$ and $N$ have similar contributions to the model output. However, they also 
have opposite effects, which may lead the inversion process to misinterpret an increase in $\theta_{i}$ as a decrease in leaf structure $N$. Therefore, in the VNIR (resp. SWIR) range, this model compensates $\theta_{i}$ underestimation (overestimation) by $N$ underestimation (overestimation).

On the other hand, several assumptions can be made regarding the good performances obtained by radiance-based inversion. First, this may be due to the noise level in the spectral measurements: as the ratio of two radiance spectra is noisier than both radiance spectra independently, retrieved pseudo-BRF spectra thus have a lower signal-to-noise ratio than originally measured radiance spectra. This problem is critical near $400 \mathrm{~nm}$ and in the NIR plateau where the sensitivity of the VNIR camera is lower compared with most of the visible range (see Fig. 4). This emphasizes the necessity of acquiring spectral measurements as clean as possible. Alternatively, such differences in the inversion performances may be due to the numerical implementation of the inversion process (e.g., through the considered cost function, optimization algorithm or initialization). Further investigation is therefore needed to fully optimize the PROCOSINE inversion.

Finally, the poorer angle estimation results obtained with bamboo leaves were likely to be due to the azimuthally anisotropic roughness properties of monocotyledon leaves, that mainly originate from the longitudinal orientation of veins (Comar et al., 2012, 2014). Unlike regular surfaces for which the BRF is azimuthally symmetric, such an organized arrangement of the leaf surface leads the specular lobe not to be in the principal plane (Miesch et al., 2002). In this case, it may seem inappropriate to represent the local 
leaf inclination at the pixel level using only a single mean angle, especially because the image spatial resolution is comparable to the inter-vein distance for monocotyledons, i.e., a few hundred micrometers (Comar et al., 2014). However, other estimation results presented in this paper strongly suggest that this does not affect the retrievals of leaf biochemical properties.

\subsection{Leaf parameter mapping}

Hyperspectral imaging allowed us to obtain high-resolution maps of model parameters by inverting the model for each pixel. Compared with the results obtained from estimated values averaged within one or several leaf disk(s) (each of which contains several hundreds of pixels), such maps provide a deeper insight in how these effects actually affect the estimation process.

In Fig. 10 and Fig. 11, we show some estimated maps obtained using PROSPECT and the radiance-based PROCOSINE model respectively, the radiance-based inversion performing better than the pseudo-BRF inversion as seen in Section 4.2. The considered bay laurel leaf was chosen due to its strong non Lambertian behavior and to the presence of a large range of $C_{a b}$ and $C_{b p}$ values. The actual chlorophyll content in the greenest area was close to $40 \mu \mathrm{g} . \mathrm{cm}^{-2}$, while the equivalent water thickness and leaf mass per area were $0.0088 \mathrm{~cm}$ and $0.0099 \mathrm{~g} . \mathrm{cm}^{-2}$ respectively.

On the one hand, as observed in Fig. 10, PROSPECT estimation was strongly affected by BRDF effects both in the VNIR and SWIR ranges. This increase in reflectance is not taken into account by PROSPECT, which resulted in some compensations during the inversion, here corresponding to an increase in $C_{b p}$ and decreases in $C_{a b}$ (as already mentioned in Section 4.2.2), $C_{w}$ and $C_{m}$. On the other hand, in Fig. 11, we show that these BRDF effects were 
(a)

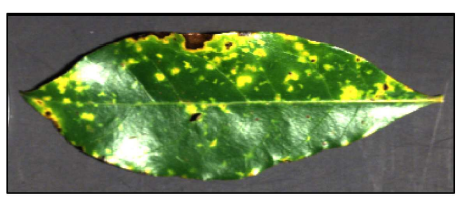

(b)

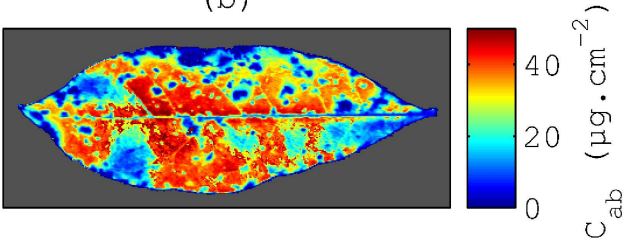

(c)

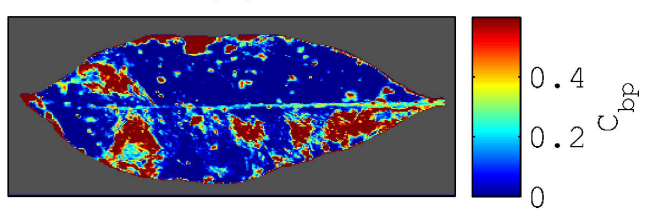

(d)

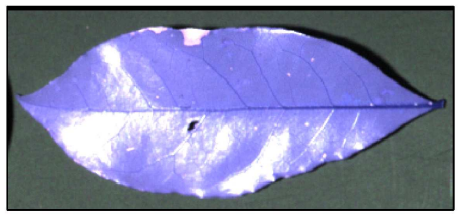

(e)
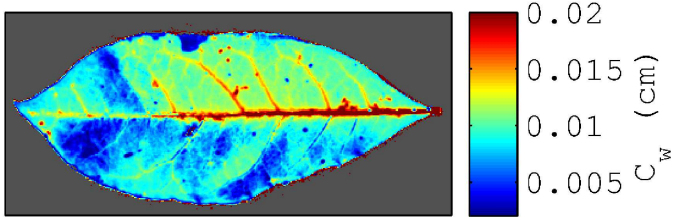

(f)

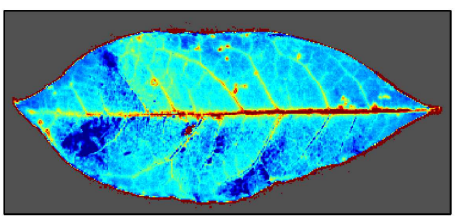

0.015 '

0.01 क्ष

0.005

Figure 10: Estimated maps obtained using PROSPECT in the VNIR (a-c) and SWIR (d-f) ranges: (a) true color composite image, (b) $C_{a b}$, (c) $C_{b p}$, (d) false color composite image (using bands $1458 \mathrm{~nm}, 2202 \mathrm{~nm}$ and $1662 \mathrm{~nm}$ ), (e) $C_{w}$, (f) $C_{m}$.

correctly interpreted as local increases in $b_{\text {spec }}$ within the PROCOSINE inversion. Accurate descriptions of the actual regular leaf orientation were also obtained through homogeneous $\theta_{i}$ maps and average $\theta_{i}$ of $15^{\circ}$ and $30^{\circ}$ for the VNIR and SWIR ranges respectively.

Modeling such extra variability therefore allowed us to obtain consistent maps of foliar content. In the VNIR range, the highest $C_{a b}$ values were obtained in the greenest parts with values close to $40 \mu \mathrm{g} . \mathrm{cm}^{-2}$, while very low chlorophyll contents were retrieved in senescent parts, especially in the necrotic area on the top (Fig. 11.b). This latter area was also exhibiting a high content in brown pigments as shown in Fig. 11.c, thereby proving the reliability 
Author-produced version of the article published in Remote Sensing of Environment, 2016, N¹77, p. 220-236.

The original publication is available at http://www.sciencedirect.com

Doi: 10.1016/j.rse.2016.02.029

(a)

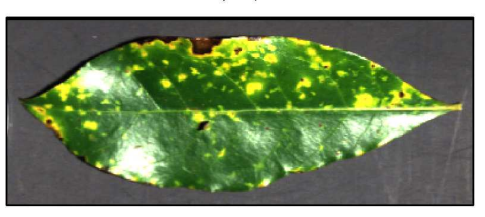

(b)

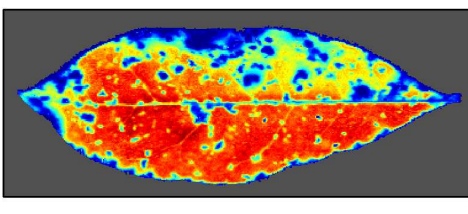

(c)

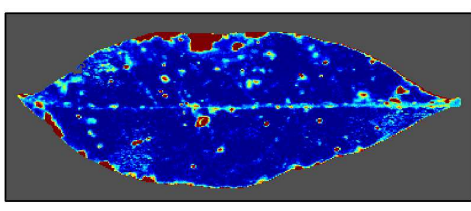

(d)

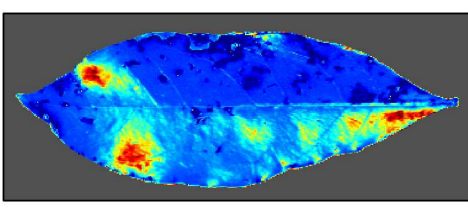

(e)

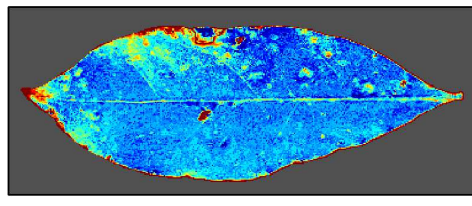

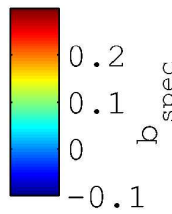
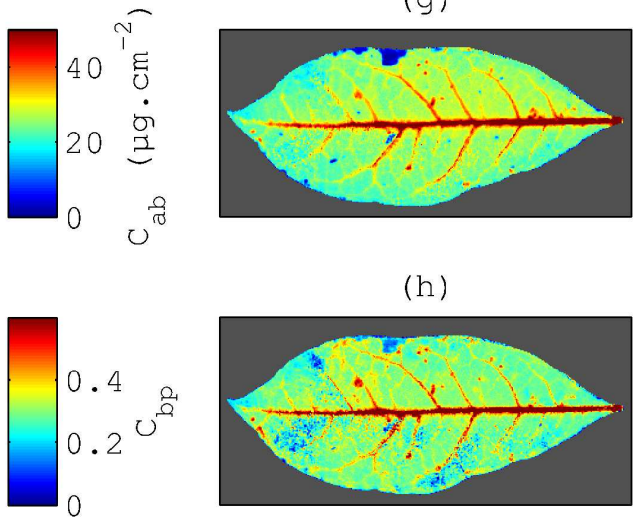

(i)

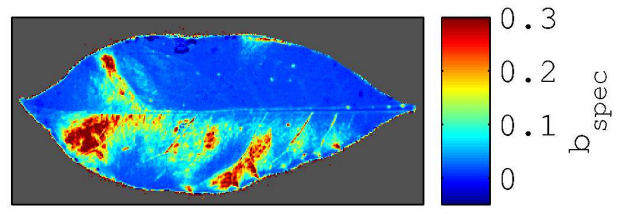

(j)

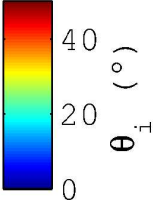

(h)

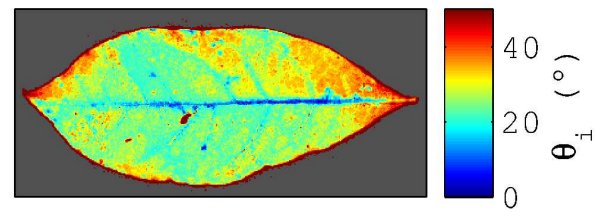

(f)

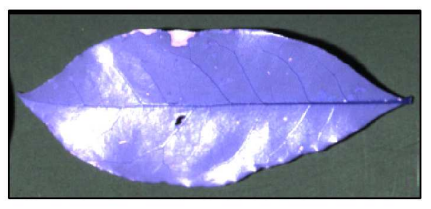

( $g$ )

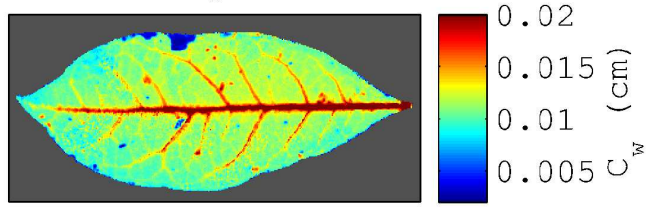

Figure 11: Estimated maps obtained using the radiance-based PROCOSINE model in the VNIR (a-e) and SWIR (f-j) ranges: (a) true color composite image, (b) $C_{a b}$, (c) $C_{b p}$, (d) $b_{\text {spec }}$, (e) $\theta_{i}$, (f) false color composite image (using bands $1458 \mathrm{~nm}, 2202 \mathrm{~nm}$ and $1662 \mathrm{~nm}$ ), (g) $C_{w},(\mathrm{~h}) C_{m},(\mathrm{i}) b_{\text {spec }},(\mathrm{j}) \theta_{i}$. 
of PROSPECT for retrieving pigments.

Consistent maps were obtained in the SWIR range as well. As expected, veins were found to contain a lot of water, unlike the upper part where necrosis was causing a strong water loss (Fig. 11.g). Although being a bit more affected than $C_{w}$ by mismodeled BRDF effects, $C_{m}$ was estimated more accurately than $C_{w}$ since both $C_{w}$ and $C_{m}$ average estimated values were close to 0.011 g.cm ${ }^{-2}$.

In particular, these maps are very appealing for identifying plant diseases at leaf scale. As described by Mahlein et al. (2013), different diseases may lead to different symptoms that can be characterized by local changes in optical properties, e.g., a higher chlorophyll/carotenoid ratio for sugar beet rust, or necrosis for Cercospora leaf spot. Therefore, inverting PROCOSINE based on VNIR and SWIR hyperspectral images of the same leaf offers very interesting perspectives for early detection of many plant diseases.

Finally, in Fig. 12, we provide a last example that again demonstrates the potential of PROCOSINE in the SWIR range when tested with a sugar beet leaf characterized by high $C_{w}\left(C_{w}=0.0355 \mathrm{~cm}\right)$ and low $C_{m}\left(C_{m}=0.0040 \mathrm{~g} . \mathrm{cm}^{-2}\right)$ values, and by a strongly non-regular and non-Lambertian leaf surface. Even if PROSPECT obtained better results when considering estimated values averaged over the five sampled leaf disks $\left(\widehat{C}_{w}=0.0370 \mathrm{~cm} ; \widehat{C}_{m}=0.0032 \mathrm{~g} . \mathrm{cm}^{-2}\right)$, it also led to highly heterogeneous and inconsistent maps, whose variances obviously did not reflect the actual ones but rather expressed unmodelled variability. Conversely, the PROCOSINE inversion led to homogeneous and consistent maps, either for leaf structure $(\widehat{N}=1.30$; to compare with 
Author-produced version of the article published in Remote Sensing of Environment, 2016, N¹77, p. 220-236.

The original publication is available at http://www.sciencedirect.com

Doi: 10.1016/j.rse.2016.02.029

(a)
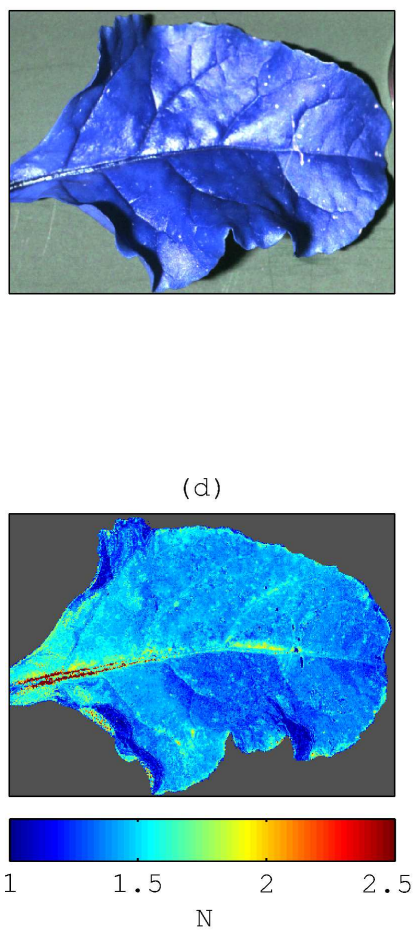

$(g)$
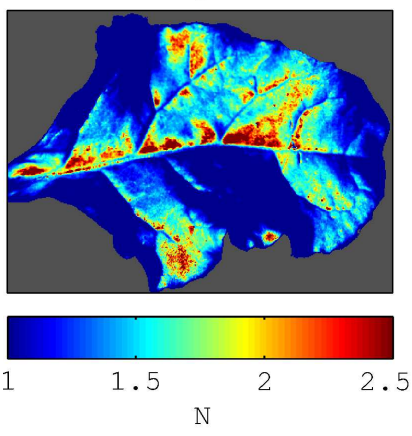

(b)
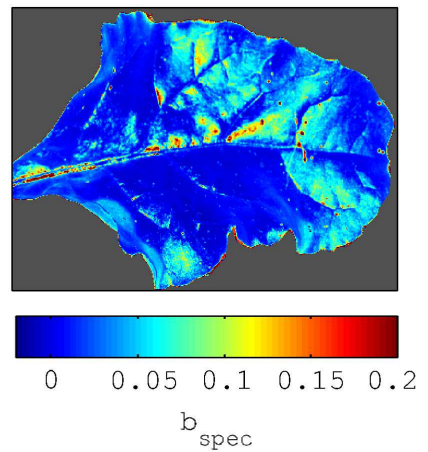

(e)
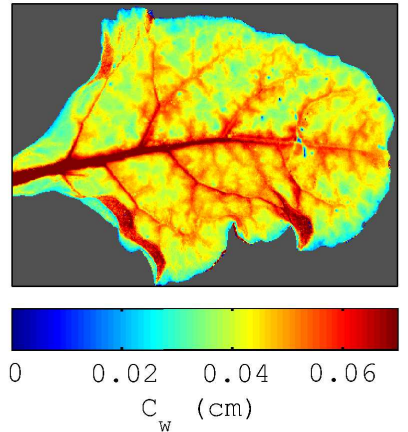

(h)

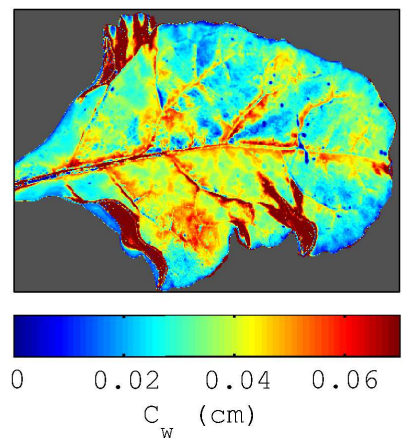

(c)
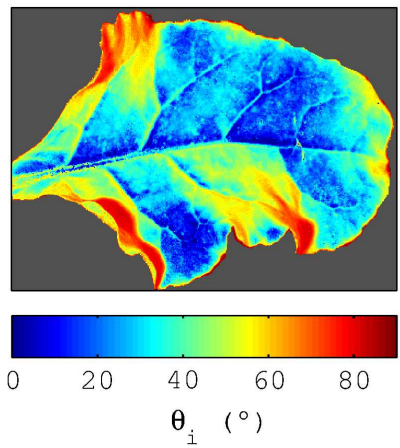

(f)

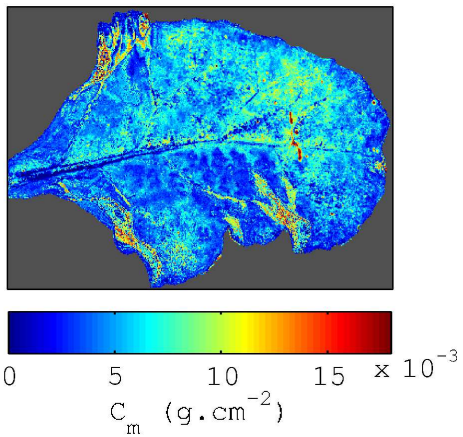

(i)

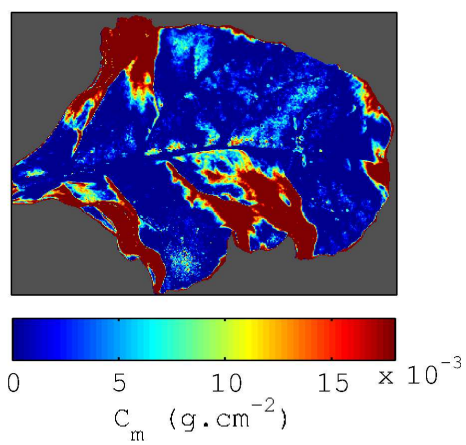

Figure 12: Estimated maps obtained in the SWIR range using the radiance-based PROCOSINE model (b-f) and PROSPECT (g-i): (a) false color composite image (using bands $1458 \mathrm{~nm}, 2202 \mathrm{~nm}$ and $1662 \mathrm{~nm}$ ), (b) $b_{s p e c}$, (c) $\theta_{i}$, (d) $N$, (e) $C_{w}$, (f) $C_{m}$, (g) $N$, (h) $C_{w}$, (i) $C_{m}$. 
the mean value of 1.225 provided by Jacquemoud et al. (1996) for the same species), equivalent water thickness $\left(\widehat{C}_{w}=0.042 \mathrm{~cm}\right)$ and leaf mass per area $\left(\widehat{C}_{m}=0.0052 \mathrm{~g} . \mathrm{cm}^{-2}\right)$. In particular, the strongly non regular leaf orientation was greatly retrieved through the estimated $\theta_{i}$ map (Fig. 12.c): regions with low estimated $\theta_{i}$ were indeed facing the light source while regions with high estimated $\theta_{i}$ were facing the opposite side (note that shadows were interpreted as high $\theta_{i}$ values). Interestingly, most imaging techniques allowing the retrieval of leaf (or even plant) orientation are either based on depth imaging systems (Chéné et al., 2012) or on 3D models built from multi-angular observations using stereovision (Lati et al., 2013) or photogrammetry (Jay et al., 2015). In comparison, it is worth noting that in this study, the retrieval of leaf orientation only necessitates a single-angular observation and is based on spectral information only.

\section{Conclusions and perspectives}

In this study, we propose a physically-based model that allows for applying rigorously a DHR model of leaf optical properties to pseudo-BRF hyperspectral (or multispectral) images acquired with close-range imaging spectroscopy. The proposed COSINE model describes the spectral variability caused by variable BRDF effects and leaf orientation, that, depending on leaf surface, can make the acquired pseudo-BRF measurements very different from DHR measurements. In this paper, COSINE is coupled with PROSPECT and the numerical inversion of the resulting PROCOSINE model led to accurate leaf-level mappings of foliar content and above leaf surface properties, both in the VNIR and SWIR ranges. 
(a)

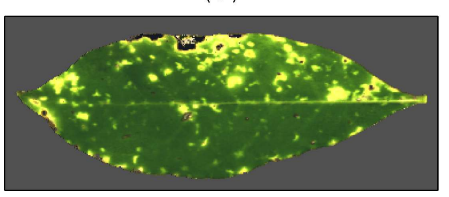

(b)

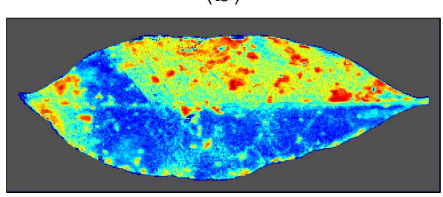

(c)

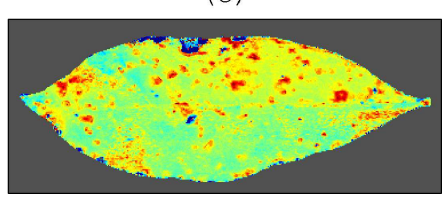

Figure 13: Use of the PROCOSINE model for image pretreatment in the VNIR (a-c) range: (a) DHR image reconstructed with PROSPECT and estimated parameters, (b) $S B R I$ map (Mahlein et al., 2013) obtained with the original image, and (c) SBRI map (Mahlein et al., 2013) obtained with the reconstructed image.

Besides avoiding the need for hemispherical measurements, the proposed approach allows the non-destructive biochemical characterization of small leaves thanks to the sub-millimeter resolution of hyperspectral images. Potentially, it could represent a practical solution for optical characterization of needles, whose optical properties were found to be well described by PROSPECT (Moorthy et al., 2008) (usual needle optical measurements involving using complex experimental setups to handle irregularities in size, shape and curvature). As previously mentioned, it can also be combined with other leaf DHR models such as LIBERTY (Dawson et al., 1998).

The accurate retrieval of $C_{m}$ makes it possible to derive mass-based quantities from surface-based quantities. In particular, strong correlations $\left(\mathrm{R}^{2}>0.85\right)$ were obtained for the estimation of Gravimetric Water Content (being expressed as $\left.C_{w} / C_{m}\right)$, which is an important indicator for fire risk modeling (Chuvieco et al., 2004).

Potential applications also include the use of PROCOSINE as a pretreatment for further processing. To illustrate, in Fig. 13.a, we show a DHR image reconstructed by running PROSPECT in a forward mode with parameters previously estimated with the radiance-based PROCOSINE model 
(the original image is presented in Fig. 10). For example, computing spectral indices designed for plant disease identification based on this cleaned image is much more reliable to detect these diseases at early infection stages, especially when these indices are based on wavelengths strongly affected by BRDF effects, e.g., the SBRI (Mahlein et al., 2013). Alternatively, PROCOSINE could allow the design of spectral indices adapted to close-range imaging spectroscopy, similarly to Féret et al. (2011).

Further investigation is however needed to properly model the specular component in the SWIR range. In this case, the effect of water absorption on leaf refractive index should be considered to reliably model the wavelengthdependency of $b_{\text {spec }}$ parameter. Also, confounding effects between PROCOSINE parameters (e.g., $N, \theta_{i}$ and $C_{m}$ in the VNIR range) should be studied more deeply by optimizing the numerical implementation of the iterative optimization or by considering regularization strategies as reviewed by Baret \& Buis (2008) and Verrelst et al. (2015), e.g., by using prior knowledge to constrain some model variables, or by generating a look-up table and averaging the $\mathrm{N}$ best solutions. As noted in the discussion, a more exhaustive assessment of PROCOSINE should also be performed over a larger data set encompassing a wider range of pigment, water and dry matter contents properly measured using laboratory extraction methods. This would allow us to fully validate the proposed model. Lastly, it is worth mentioning that the use of COSINE based on images acquired under outdoor conditions requires assuming that diffuse illumination (coming from either the sky and/or the surrounding terrain) is negligible compared with direct sunlight. We are currently working towards an improvement of COSINE to account for these 
influences within the modeling, thus transforming the BRF-based model into a hemispherical-directional reflectance factor based model. This would allow processing spatially-resolved data acquired from near-ground remote sensing, e.g., from a tower or low flying unmanned aerial vehicle.

\section{Acknowledgments}

This study was funded by the French National Research Agency, within the program "Investissements d'avenir" with the reference ANR-11-BTBR0007 (AKER project). Many thanks to Xavier Briottet and the anonymous reviewers for their valuable comments and suggestions.

\section{References}

Ashikmin, M., Premože, S., \& Shirley, P. (2000). A microfacet-based BRDF generator. In Proceedings of the 27th annual conference on Computer graphics and interactive techniques (pp. 65-74). ACM Press/AddisonWesley Publishing Co.

Bacour, C., Jacquemoud, S., Tourbier, Y., Dechambre, M., \& Frangi, J.-P. (2002). Design and analysis of numerical experiments to compare four canopy reflectance models. Remote Sensing of Environment, 79, 72-83.

Bacour, C., Jacquemoud, S., Vogt, P., Hosgood, B., Andreoli, G., \& Frangi, J.-p. (2001). Optimal sampling configurations for the estimation of canopy properties from BRDF data acquired with the EGO/JRC. In Proc. 8th International Symposium Physical Measurements 86 Signatures in Remote Sensing (pp. 481-486). 
Baret, F., \& Buis, S. (2008). Estimating canopy characteristics from remote sensing observations: Review of methods and associated problems. In S. Liang (Ed.), Advances in Land Remote Sensing (pp. 173-201). Springer Netherlands.

Baret, F., \& Fourty, T. (1997a). Estimation of leaf water content and specific leaf weight from reflectance and transmittance measurements. Agronomie, $17,455-464$.

Baret, F., \& Fourty, T. (1997b). Radiometric estimates of nitrogen status of leaves and canopies. In G. Lemaire (Ed.), Diagnosis of the Nitrogen Status in Crops (pp. 201-227). Springer Berlin Heidelberg.

Blackburn, G. A. (2007). Hyperspectral remote sensing of plant pigments. Journal of experimental botany, 58, 855-867.

Born, M., \& Wolf, E. (1980). Chapter XIII - Optics of metals. In M. Born, \& E. Wolf (Eds.), Principles of Optics (pp. 611 - 664). Pergamon. (Sixth (corrected) edition ed.).

Bousquet, L., Lachérade, S., Jacquemoud, S., \& Moya, I. (2005). Leaf BRDF measurements and model for specular and diffuse components differentiation. Remote Sensing of Environment, 98, 201-211.

Bowyer, P., \& Danson, F. (2004). Sensitivity of spectral reflectance to variation in live fuel moisture content at leaf and canopy level. Remote Sensing of Environment, 92, 297-308.

Buddenbaum, H., \& Hill, J. (2015). PROSPECT inversions of leaf laboratory 
imaging spectroscopy-a comparison of spectral range and inversion technique influences. Photogrammetrie-Fernerkundung-Geoinformation, 2015, $231-240$.

Cerovic, Z. G., Masdoumier, G., Ghozlen, N. B., \& Latouche, G. (2012). A new optical leaf-clip meter for simultaneous non-destructive assessment of leaf chlorophyll and epidermal flavonoids. Physiologia plantarum, 146, $251-260$

Chen, J., \& Leblanc, S. (1997). A four-scale bidirectional reflectance model based on canopy architecture. Geoscience and Remote Sensing, IEEE Transactions on, 35, 1316-1337.

Chéné, Y., Rousseau, D., Lucidarme, P., Bertheloot, J., Caffier, V., Morel, P., Belin, E., \& Chapeau-Blondeau, F. (2012). On the use of depth camera for 3D phenotyping of entire plants. Computers and Electronics in Agriculture, 82, 122-127.

Cheng, T., Rivard, B., \& Sanchez-Azofeifa, A. (2011). Spectroscopic determination of leaf water content using continuous wavelet analysis. Remote Sensing of Environment, 115, 659-670.

Chuvieco, E., Cocero, D., Riano, D., Martin, P., Martmez-Vega, J., de la Riva, J., \& Pérez, F. (2004). Combining NDVI and surface temperature for the estimation of live fuel moisture content in forest fire danger rating. Remote Sensing of Environment, 92, 322-331.

Colombo, R., Meroni, M., Marchesi, A., Busetto, L., Rossini, M., Giardino, C., \& Panigada, C. (2008). Estimation of leaf and canopy water content in 
poplar plantations by means of hyperspectral indices and inverse modeling. Remote Sensing of Environment, 112, 1820 - 1834.

Comar, A., Baret, F., Obein, G., Simonot, L., Meneveaux, D., Vinot, F., \& de Solan, B. (2014). ACT: A leaf BRDF model taking into account the azimuthal anisotropy of monocotyledonous leaf surface. Remote Sensing of Environment, 143, 112 - 121.

Comar, A., Baret, F., Vinot, F., Yan, L., \& de Solan, B. (2012). Wheat leaf bidirectional reflectance measurements: Description and quantification of the volume, specular and hot-spot scattering features. Remote Sensing of Environment, 121, $26-35$.

Curran, P. J. (1989). Remote sensing of foliar chemistry. Remote Sensing of Environment, 30, $271-278$.

Danson, F., \& Bowyer, P. (2004). Estimating live fuel moisture content from remotely sensed reflectance. Remote Sensing of Environment, 92, 309-321.

Dawson, T. P., Curran, P. J., \& Plummer, S. E. (1998). LIBERTY - modeling the effects of leaf biochemical concentration on reflectance spectra. Remote Sensing of Environment, 65, 50-60.

Dorigo, W., Zurita-Milla, R., de Wit, A., Brazile, J., Singh, R., \& Schaepman, M. (2007). A review on reflective remote sensing and data assimilation techniques for enhanced agroecosystem modeling. International Journal of Applied Earth Observation and Geoinformation, 9, 165- 193. 
Féret, J.-B., \& Asner, G. P. (2014). Mapping tropical forest canopy diversity using high-fidelity imaging spectroscopy. Ecological Applications, 24, $1289-1296$.

Féret, J.-B., François, C., Asner, G. P., Gitelson, A. A., Martin, R. E., Bidel, L. P., Ustin, S. L., le Maire, G., \& Jacquemoud, S. (2008). PROSPECT-4 and 5: Advances in the leaf optical properties model separating photosynthetic pigments. Remote Sensing of Environment, 112, 3030-3043.

Féret, J.-B., François, C., Gitelson, A., Asner, G. P., Barry, K. M., Panigada, C., Richardson, A. D., \& Jacquemoud, S. (2011). Optimizing spectral indices and chemometric analysis of leaf chemical properties using radiative transfer modeling. Remote sensing of environment, 115, 2742-2750.

Gastellu-Etchegorry, J., Demarez, V., Pinel, V., \& Zagolski, F. (1996). Modeling radiative transfer in heterogeneous $3-\mathrm{D}$ vegetation canopies. Remote Sensing of Environment, 58, $131-156$.

Gerber, F., Marion, R., Olioso, A., Jacquemoud, S., da Luz, B. R., \& Fabre, S. (2011). Modeling directional-hemispherical reflectance and transmittance of fresh and dry leaves from $0.4 \mu \mathrm{m}$ to $5.7 \mu \mathrm{m}$ with the PROSPECTVISIR model. Remote Sensing of Environment, 115, 404-414.

Hale, G. M., \& Querry, M. R. (1973). Optical constants of water in the 200-nm to 200- $\mu \mathrm{m}$ wavelength region. Appl. Opt., 12, 555-563.

Hochberg, E. J., Andrefouet, S., \& Tyler, M. R. (2003). Sea surface correction of high spatial resolution Ikonos images to improve bottom mapping 
in near-shore environments. Geoscience and Remote Sensing, IEEE Transactions on, 41, 1724-1729.

Jacquemoud, S., Bacour, C., Poilv, H., \& Frangi, J.-P. (2000). Comparison of four radiative transfer models to simulate plant canopies reflectance: Direct and inverse mode. Remote Sensing of Environment, 74, 471 - 481.

Jacquemoud, S., \& Baret, F. (1990). PROSPECT: A model of leaf optical properties spectra. Remote sensing of environment, 34, 75-91.

Jacquemoud, S., Ustin, S., Verdebout, J., Schmuck, G., Andreoli, G., \& Hosgood, B. (1996). Estimating leaf biochemistry using the PROSPECT leaf optical properties model. Remote Sensing of Environment, 56, 194 202.

Jacquemoud, S., Verhoef, W., Baret, F., Bacour, C., Zarco-Tejada, P. J., Asner, G. P., François, C., \& Ustin, S. L. (2009). PROSPECT+SAIL models: A review of use for vegetation characterization. Remote Sensing of Environment, 113, S56 - S66.

Jay, S., Hadoux, X., Gorretta, N., \& Rabatel, G. (2014). Potential of hyperspectral imagery for nitrogen content retrieval in sugar beet leaves. In Proc. Int. Conf. Ag. Eng., AgEng2014, Zurich. The European Society of Agricultural Engineers (EurAgEng).

Jay, S., Rabatel, G., Hadoux, X., Moura, D., \& Gorretta, N. (2015). In-field crop row phenotyping from 3D modeling performed using Structure from Motion. Computers and Electronics in Agriculture, 110, 70-77. 
Ji-Yong, S., Xiao-Bo, Z., Jie-Wen, Z., Kai-Liang, W., Zheng-Wei, C., XiaoWei, H., De-Tao, Z., \& Holmes, M. (2012). Nondestructive diagnostics of nitrogen deficiency by cucumber leaf chlorophyll distribution map based on near infrared hyperspectral imaging. Scientia horticulturae, 138, 190-197.

Knyazikhin, Y., Schull, M. a., Stenberg, P., Mõttus, M., Rautiainen, M., Yang, Y., Marshak, A., Latorre Carmona, P., Kaufmann, R. K., Lewis, P., Disney, M. I., Vanderbilt, V., Davis, A. B., Baret, F., Jacquemoud, S., Lyapustin, A., \& Myneni, R. B. (2013). Hyperspectral remote sensing of foliar nitrogen content. Proceedings of the National Academy of Sciences, 110, E185-E192.

Lati, R. N., Filin, S., \& Eizenberg, H. (2013). Estimating plant growth parameters using an energy minimization-based stereovision model. Computers and Electronics in Agriculture, 98, 260-271.

Mahlein, A.-K., Rumpf, T., Welke, P., Dehne, H.-W., Plümer, L., Steiner, U., \& Oerke, E.-C. (2013). Development of spectral indices for detecting and identifying plant diseases. Remote Sensing of Environment, 128, 21-30.

Miesch, C., Briottet, X., \& Kerr, Y. (2002). Bidirectional reflectance of a rough anisotropic surface. International Journal of Remote Sensing, 23, $3107-3114$.

Moorthy, I., Miller, J. R., \& Noland, T. L. (2008). Estimating chlorophyll concentration in conifer needles with hyperspectral data: An assessment at the needle and canopy level. Remote Sensing of Environment, 112, $2824-2838$ 
Nicodemus, F. E., Richmond, J. C., Hsia, J. J., Ginsberg, I. W., \& Limperis, T. (1977). Geometrical considerations and nomenclature for reflectance volume 160. US Department of Commerce, National Bureau of Standards Washington, DC, USA.

Nicotra, A., Hofmann, M., Siebke, K., \& Ball, M. (2003). Spatial patterning of pigmentation in evergreen leaves in response to freezing stress. Plant, Cell \& Environment, 26, 1893-1904.

Saltelli, A., Tarantola, S., \& Chan, K.-S. (1999). A quantitative modelindependent method for global sensitivity analysis of model output. Technometrics, 41, 39-56.

Schaepman-Strub, G., Schaepman, M., Painter, T., Dangel, S., \& Martonchik, J. (2006). Reflectance quantities in optical remote sensing - definitions and case studies. Remote sensing of environment, 103, 27-42.

Schlemmer, M., Gitelson, A., Schepers, J., Ferguson, R., Peng, Y., Shanahan, J., \& Rundquist, D. (2013). Remote estimation of nitrogen and chlorophyll contents in maize at leaf and canopy levels. International Journal of Applied Earth Observation and Geoinformation, 25, 47 - 54 .

Stuckens, J., Somers, B., Delalieux, S., Verstraeten, W., \& Coppin, P. (2009). The impact of common assumptions on canopy radiative transfer simulations: A case study in citrus orchards. Journal of Quantitative Spectroscopy and Radiative Transfer, 110, 1-21.

Ustin, S. L., Gitelson, A. A., Jacquemoud, S., Schaepman, M., Asner, G. P., Gamon, J. A., \& Zarco-Tejada, P. (2009). Retrieval of foliar information 
about plant pigment systems from high resolution spectroscopy. Remote Sensing of Environment, 113, S67-S77.

Vanderbilt, V. C., \& Grant, L. (1985). Plant canopy specular reflectance model. Geoscience and Remote Sensing, IEEE Transactions on, (pp. 722$730)$.

Verhoef, W. (1984). Light scattering by leaf layers with application to canopy reflectance modeling: The SAIL model. Remote Sensing of Environment, $16,125-141$.

Verrelst, J., Camps-Valls, G., Muñoz-Marí, J., Rivera, J. P., Veroustraete, F., Clevers, J. G., \& Moreno, J. (2015). Optical remote sensing and the retrieval of terrestrial vegetation bio-geophysical properties-a review. ISPRS Journal of Photogrammetry and Remote Sensing, in press, .

Vigneau, N., Ecarnot, M., Rabatel, G., \& Roumet, P. (2011). Potential of field hyperspectral imaging as a non destructive method to assess leaf nitrogen content in wheat. Field Crops Research, 122, 25-31.

Wallach, D., Makowski, D., Jones, J. W., \& Brun, F. (2014). Working with dynamic crop models. Methods, tools and examples for agriculture and environment. Londres, GBR: Academic Press - Elsevier.

Zarco-Tejada, P. J., Miller, J., Morales, A., Berjón, A., \& Aguera, J. (2004). Hyperspectral indices and model simulation for chlorophyll estimation in open-canopy tree crops. Remote Sensing of Environment, 90, 463-476.

Zarco-Tejada, P. J., Rueda, C., \& Ustin, S. (2003). Water content estimation 
in vegetation with MODIS reflectance data and model inversion methods.

Remote Sensing of Environment, 85, 109-124.

\section{List of Figures}

1 Angle representation. . . . . . . . . . . . . . . . . 9

2 Experimental setup for the horizontal (a) and tilted (b-c) configurations. For the VNIR camera, the corresponding average incident angles $\bar{\theta}_{i}$ are $20^{\circ}$ (a), $40^{\circ}$ (b) and $0^{\circ}$ (c). For the SWIR camera, the corresponding average incident angles $\bar{\theta}_{i}$ are $30^{\circ}$ (a), $10^{\circ}$ (b) and $50^{\circ}$ (c). . . . . . . . . . . . 17

3 First-order sensitivity indices and interactions for (a) PROSPECT in the VNIR range, (b) PROSPECT in the SWIR range, (c) PROCOSINE in the VNIR range, and (d) PROCOSINE in the SWIR range $\left(\theta_{s}=26^{\circ}\right) . \ldots \ldots \ldots$

4 Fitting results obtained for the VNIR range (a-c) and the SWIR range (d-f) using the pseudo-BRF model (b,e) and the radiance model $(c, f)$. Solid (resp. dashed) lines correspond to measurements (resp. simulations). Blue curves correspond to areas strongly affected by specular reflection, while red curves correspond to areas characterized by a high incident angle. . . 30 
$5 C_{a b}$ estimation results (mean $+/$ - standard deviation) obtained with PROSPECT (blue), the pseudo-BRF based PROCOSINE model (orange), and the radiance-based PROCOSINE model (green) and with $\bar{\theta}_{i}=20^{\circ}$ (a-c), $\bar{\theta}_{i}=0^{\circ}$ (d-f) and $\bar{\theta}_{i}=40^{\circ}(\mathrm{g}-\mathrm{i})$. The $\mathrm{R}^{2}$ and RMSE values are computed for actual $C_{a b}$ values lower than $40 \mu \mathrm{g} . \mathrm{cm}^{-2}$ and are given in paren-

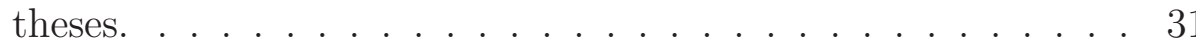

6 Histograms of estimated $\theta_{i}$ values for every pixel for the VNIR range and the three acquisition configurations $\left(\bar{\theta}_{i}=0^{\circ}, \bar{\theta}_{i}=\right.$ $20^{\circ}$, and $\bar{\theta}_{i}=40^{\circ}$ ): (a) pseudo-BRF based PROCOSINE model, and (b) radiance-based PROCOSINE model. . . . . . . 33

$7 \quad C_{w}$ estimation results (mean $+/$ - standard deviation) obtained with PROSPECT (blue), the pseudo-BRF based PROCOSINE model (orange), and the radiance-based PROCOSINE model (green) and with $\bar{\theta}_{i}=30^{\circ}$ (a-c), $\bar{\theta}_{i}=10^{\circ}$ (d-f) and $\bar{\theta}_{i}=50^{\circ}$ (g-i). $\mathrm{R}^{2}$ and RMSE values are given in parentheses. . 35

$8 C_{m}$ estimation results (mean $+/$ - standard deviation) obtained with PROSPECT (blue), the pseudo-BRF based PROCOSINE model (orange), and the radiance-based PROCOSINE model (green) and with $\bar{\theta}_{i}=30^{\circ}$ (a-c), $\bar{\theta}_{i}=10^{\circ}(\mathrm{d}-\mathrm{f})$ and $\bar{\theta}_{i}=50^{\circ}(\mathrm{g}-\mathrm{i}) . \mathrm{R}^{2}$ and RMSE values are given in parentheses. . 36

9 Histograms of estimated $\theta_{i}$ values in every pixel for the SWIR range and the three acquisition configurations $\left(\bar{\theta}_{i}=10^{\circ}, \bar{\theta}_{i}=\right.$ $30^{\circ}$, and $\bar{\theta}_{i}=50^{\circ}$ ): (a) pseudo-BRF based PROCOSINE model, and (b) radiance-based PROCOSINE model. . . . . . . 37 
10 Estimated maps obtained using PROSPECT in the VNIR (ac) and SWIR (d-f) ranges: (a) true color composite image, (b) $C_{a b}$, (c) $C_{b p}$, (d) false color composite image (using bands $1458 \mathrm{~nm}, 2202 \mathrm{~nm}$ and $1662 \mathrm{~nm}$ ), (e) $C_{w}$, (f) $C_{m} \ldots \ldots 44$

11 Estimated maps obtained using the radiance-based PROCOSINE model in the VNIR (a-e) and SWIR (f-j) ranges: (a) true color composite image, (b) $C_{a b}$, (c) $C_{b p}$, (d) $b_{\text {spec }}$, (e) $\theta_{i}$, (f) false color composite image (using bands $1458 \mathrm{~nm}, 2202 \mathrm{~nm}$ and $1662 \mathrm{~nm}),(\mathrm{g}) C_{w}$, (h) $C_{m}$, (i) $b_{\text {spec }},(\mathrm{j}) \theta_{i} \ldots \ldots \ldots$

12 Estimated maps obtained in the SWIR range using the radiancebased PROCOSINE model (b-f) and PROSPECT (g-i): (a) false color composite image (using bands $1458 \mathrm{~nm}, 2202 \mathrm{~nm}$ and $1662 \mathrm{~nm}$ ), (b) $b_{\text {spec }}$, (c) $\theta_{i}$, (d) $N$, (e) $C_{w}$, (f) $C_{m}$, (g) $N$, (h) $C_{w}$, (i) $C_{m} \ldots \ldots \ldots \ldots \ldots$

13 Use of the PROCOSINE model for image pretreatment in the VNIR (a-c) range: (a) DHR image reconstructed with PROSPECT and estimated parameters, (b) SBRI map (Mahlein et al., 2013) obtained with the original image, and (c) SBRI map (Mahlein et al., 2013) obtained with the reconstructed image. . . . . . . . . . . . . . . . . 49 